\title{
Models for the Configuration and Integrity of Partially Oxidized Fuel Rod Cladding at High Temperatures
}

\author{
L. J. Siefken
}

Published January 1999

Idaho National Engineering and Environmental Laboratory

" Lockheed Martin Idaho Technologies Company Idaho Falls, Idaho 83415

Prepared for the

U.S. Nuclear Regulatory Commission

Washington, D. C. 20555 


\section{DISCLAIMER}

This report was prepared as an account of work sponsored by an agency of the United States Government. Neither the United States Government nor any agency thereof, nor any of their employees, make any warranty, express or implied, or assumes any legal liability or responsibility for the accuracy, completeness, or usefulness of any information, apparatus, product, or process disclosed, or represents that its use would not infringe privately owned rights. Reference herein to any specific commercial product, process, or service by trade name, trademark, manufacturer, or otherwise does not necessarily constitute or imply its endorsement, recommendation, or favoring by the United States Government or any agency thereof. The views and opinions of authors expressed herein do not necessarily state or reflect those of the United States Government or any agency thereof. 


\section{DISCLAIMER}

Portions of this document may be illegible in electronic image products. Images are produced from the best available original document. 


\begin{abstract}
Models were designed to resolve deficiencies in the SCDAP/RELAP5/MOD3.2 calculations of the configuration and integrity of hot, partially oxidized cladding. These models are expected to improve the calculations of several important aspects of fuel rod behavior. First, an improved mapping was established from a compilation of PIE results from severe fuel damage tests of the configuration of melted metallic cladding that is retained by an oxide layer. The improved mapping accounts for the relocation of melted cladding in the circumferential direction. Then, rules based on PIE results were established for calculating the effect of cladding that has relocated from above on the oxidation and integrity of the lower intact cladding upon which it solidifies. Next, three different methods were identified for calculating the extent of dissolution of the oxidic part of the cladding due to its contact with the metallic part. The extent of dissolution effects the stress and thus the integrity of the oxidic part of the cladding. Then, an empirical equation was presented for calculating the stress in the oxidic part of the cladding and evaluating its integrity based on this calculated stress. This empirical equation replaces the current criterion for loss of integrity which is based on temperature and extent of oxidation. Finally, a new rule based on theoretical and experimental results was established for identifying the regions of a fuel rod with oxidation of both the inside and outside surfaces of the cladding. The implementation of these models is expected to eliminate the tendency of the SCDAP/ RELAP5 code to overpredict the extent of oxidation of the upper part of fuel rods and to underpredict the extent of oxidation of the lower part of fuel rods and the part with a high concentration of relocated material. This report is a revision and reissue of the report entitled, "Improvements in Modeling of Cladding Oxidation and Meltdown."
\end{abstract}




\section{CONTENTS}

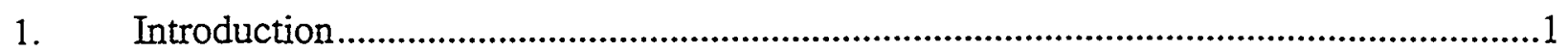

2. Assessment of Calculated Axial Distribution in Oxidation .............................................6

3. PIE Results for Mapping Cladding Configuration.........................................................12

4. Rules for Calculating Configuration and Integrity of Cladding.......................................16

4.1 Circumferential Relocation of Melted Cladding.................................................17

4.2 Behavior of Cladding at Locations with Relocated Material...............................18

4.3 Dissolution and Thinning of Oxide Layer .........................................................20

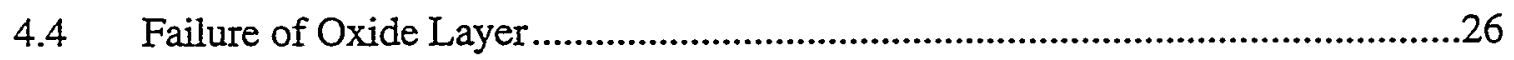

4.5 -Identification of Regions with Double-Sided Oxidation .....................................30

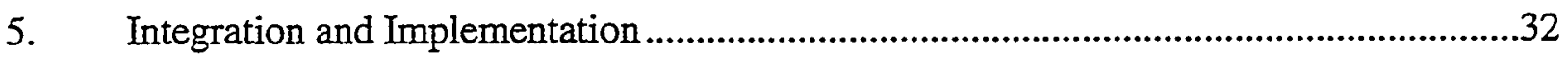

6. Testing and Assessment of Model Improvements ..........................................................35

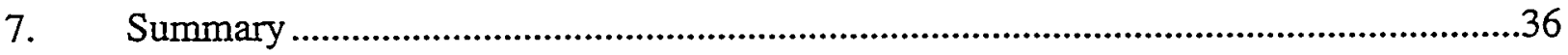

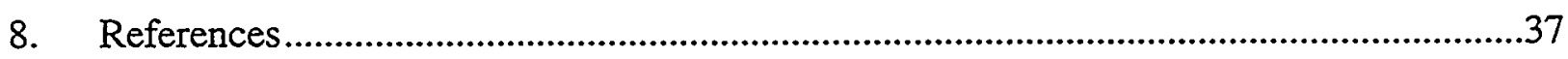

\section{FIGURES}

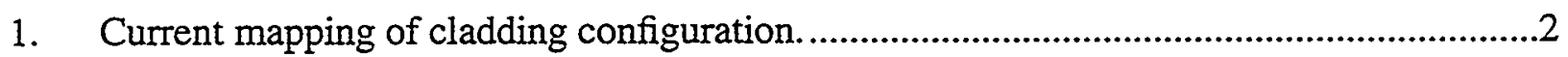

2. Mapping of cladding configuration as indicated by PIE results. ...........................................

3. Configuration of cladding for damage index " 3 "....................................................................

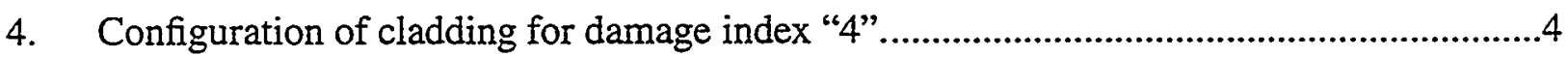

5. Configuration of cladding for damage index " 5 "..........................................................

6. Configuration of cladding for damage index " 6 "................................................................

7. Example of diverse configurations of liquefied metallic layer from PIE results of OECD LOFT FP-2 experiment ...................................................................13

8. Example of asymmetric configuration of liquefied metallic layer from $0.96 \mathrm{~m}$ elevation of OECD LOFT FP-2 Test bundle (L1 Area 1).....................................13

9. Example of asymmetric configuration of liquefied metallic layer from $0.96 \mathrm{~m}$ elevation of OECD LOFT FP-2 Test bundle (L1Area2)........................................14

10. Example of asymmetric configuration of liquefied metallic layer from 1.04 m elevation of OECD LOFT FP-2 test bundle (M3Area1) ........................................14

11. Example of asymmetric configuration of liquefied metallic layer from $1.04 \mathrm{~m}$ elevation of OECD LOFT FP-2 test bundle (M4Area1)...

12. Example of asymmetric shape of liquefied cladding and dissolved fuel from

PBF SFD 1-1 experiment.

13. Example from OECD LOFT FP-2 experiment of fully oxidized cladding surrounded by relocated material. 
14. Configuration change resulting from melting of metallic part of cladding. ......................17

15. Crust formation at bottom surface of large concentration of relocated material. ...............19

16. Cladding configuration calculated by models for dissolution and oxidation......................24

17. Schematic of change in thickness of cladding oxide layer during time step.....................24

18. Integration and application of models for cladding configuration and integrity.................33

19. Flow chart of Group A subroutines in Figure 18 .............................................................34

20. Flow chart of Group B subroutines in Figure 18 ..........................................................3

\section{TABLES}

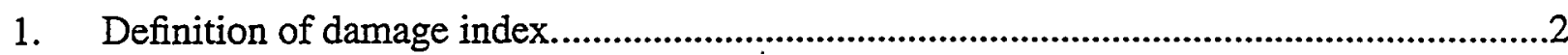

2. Comparison of calculated and measured axial distribution in cladding oxidation for PBF SFD 1-1 test.

3. Comparison of calculated and measured axial distributions in extent of cladding oxidation for PBF SFD 1-4 test...............................................................................8

4. Comparison of calculated and measured axial distributions in extent of cladding oxidation for KfK CORA-13 test.

5. Comparison of calculated and measured axial distributions in extent of cladding oxidation for PHEBUS B9+ test.

6. Comparison of calculated and measured axial distributions in extent of cladding oxidation for OECD LOFT FP-2 test.

7. Comparison of depths of dissolution of $\mathrm{UO}_{2}$ and $\mathrm{ZrO}_{2}$ for case of no prior dissolution and time interval of $0.1 \mathrm{~s}$.

8. Stress ratio in cladding oxide layer for typical range of severe accident conditions.

9. Comparison of cladding oxidation for PBF SFD 1-1 test with current and proposed models for evaluating cladding integrity.

10. Current model for identifying regions with double-sided oxidation.

11. Proposed model for identifying regions with double-sided oxidation.

12. Matrix of test problems for assessing improvements in modeling of cladding oxidation and meltdown. 


\section{Introduction}

The configuration and integrity of partially oxidized fuel rod cladding at high temperatures has a significant influence on the progression of damage in a reactor core during a severe accident. The configuration and integrity change as the cladding temperature increases. First, ballooning and rupture of the cladding may occur due to excessive internal pressure in the fuel rods. Double-sided oxidation will occur in the neighborhood of the rupture. If cladding heatup continues, the metallic part of the cladding will melt. If no cladding ballooning has occurred, the melted metallic part of the cladding remains configured as an annulus. If cladding ballooning has occurred, the metallic part of the cladding undergoes some relocation in the circumferential direction and changes into the configuration of a segment of an annulus ("quarter moon" configuration). Next, the metallic part of the cladding begins to dissolve and diminish the thickness of the oxidic part of the cladding adjacent to it. If the cladding maintains a high temperature, the oxidic part of the cladding may fail due to stresses or be diminished in thickness to the vanishing point for the case of steam-starved conditions. Then, the metallic part of the cladding slumps to a lower location. If relocated cladding material from a higher location freezes on the outside surface of the cladding, the integrity of oxidic part of the cladding may be reinforced. These changes in the configuration of the metallic part of the cladding and in the integrity of the oxidic part of the cladding have a significant impact on the size of the area over which cladding oxidation and thus on oxidation driven heatup of a reactor core.

The assessment of SCDAP/RELAP5/MOD3.2 ${ }^{1}$ indicated that the code was overpredicting the extent of oxidation of cladding in the upper part of fuel rods and underpredicting the extent of oxidation in the lower part of fuel rods. The assessment indicated that the overall oxidation of fuel rods was in good agreement with experimental results but that there was a significant discrepancy between calculated and measured axial distribution of oxidation. This discrepancy in the axial distribution of cladding oxidation is considered to be due to incorrect mapping of the configuration of the metallic part of the cladding and of the integrity of the oxidic part of the cladding as fuel rod heatup and damage progression occurs. The current mapping of the configuration of the metallic part of the cladding with respect to a damage index is shown in Figure 1. The damage index is defined in Table 1. The reacting annulus of melted cladding indicated in Figure 1 is a reference to metallic cladding that chemically reacts with the oxide layer it contacts and dissolves at least part of it. As indicated from Post-Irradiation Examinations (PIE), the correct mapping of cladding configuration with respect to damage index should be as shown in Figure 2. For damage indexes " 1 " and " 2 ", the metallic and oxidic parts of the cladding maintain a perfect annular configuration and there is no reaction of the metallic part with the oxidic part. A schematic of the cladding configuration for damage index " 3 " as it is currently modeled and as PIE results indicate it should be modeled is shown in Figure 3. The dissolution of the oxidic part of the cladding by the metallic part of the cladding is currently not calculated but modeling changes are proposed to calculate this process. Since this report does not propose changes in the modeling of fuel dissolution, this process is not shown in Figure 3. Schematics of the proposed cladding configurations for damage indices " 4 ", " 5 ", and " 6 " are shown in Figure 4, Figure 5, and Figure 6, respectively. These figures also show the current cladding configuration with respect to damage index. As shown in Figure 6, melted cladding that slumps is in the configuration of drops for both the current and the proposed modeling. 


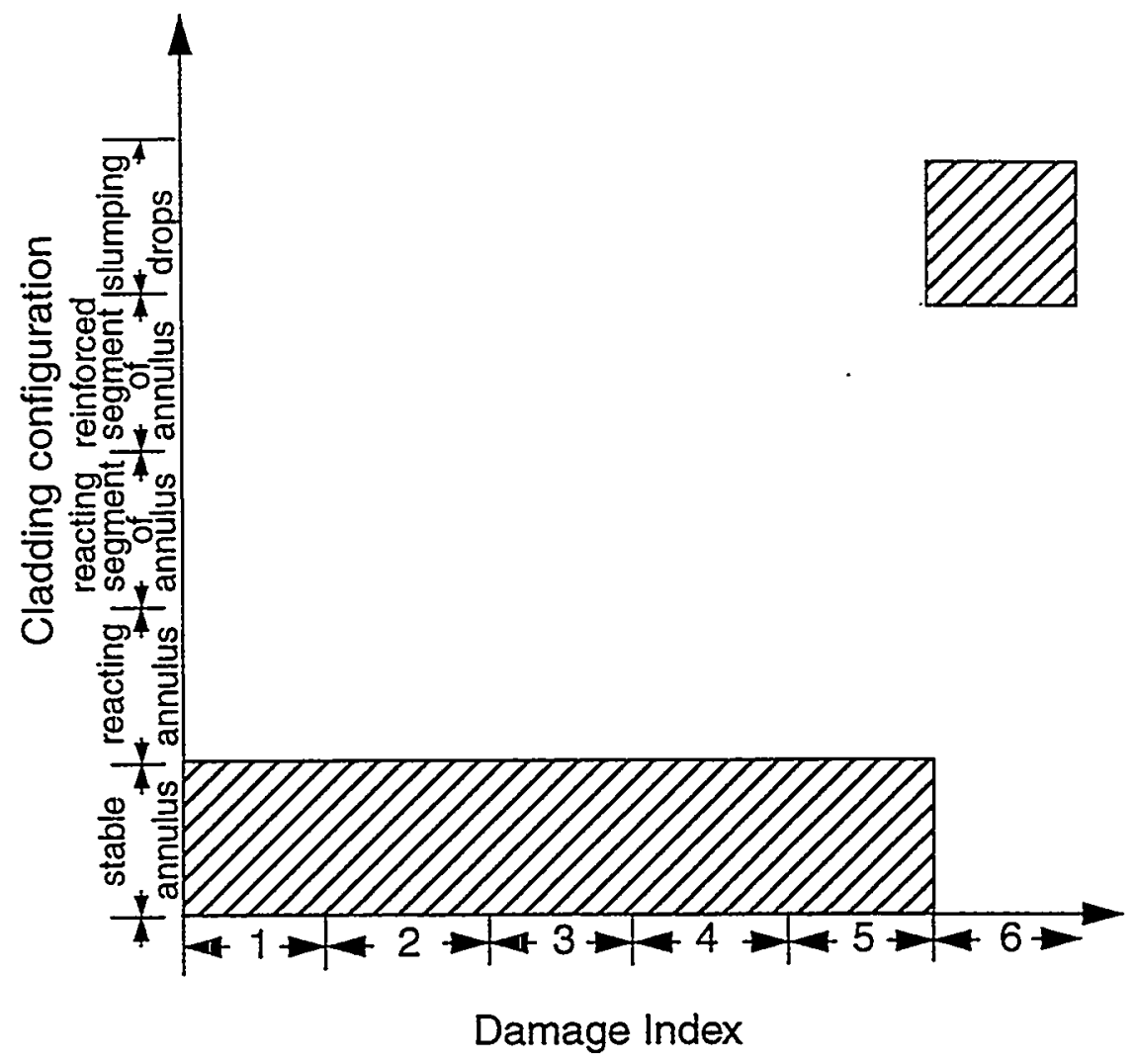

Figure 1. Current mapping of cladding configuration.

Table 1. Definition of damage index.

\begin{tabular}{|c|c|}
\hline $\begin{array}{c}\text { Damage } \\
\text { index }\end{array}$ & Definition \\
\hline \hline 1 & no cladding ballooning or melting \\
\hline 2 & cladding ballooning but no melting \\
\hline 3 & cladding melting but no ballooning \\
\hline 4 & cladding melting and ballooning \\
\hline 5 & cladding melting and ballooned or not ballooned, and presence of relocated material \\
\hline 6 & breach or vanishing of oxidic part of cladding \\
\hline
\end{tabular}




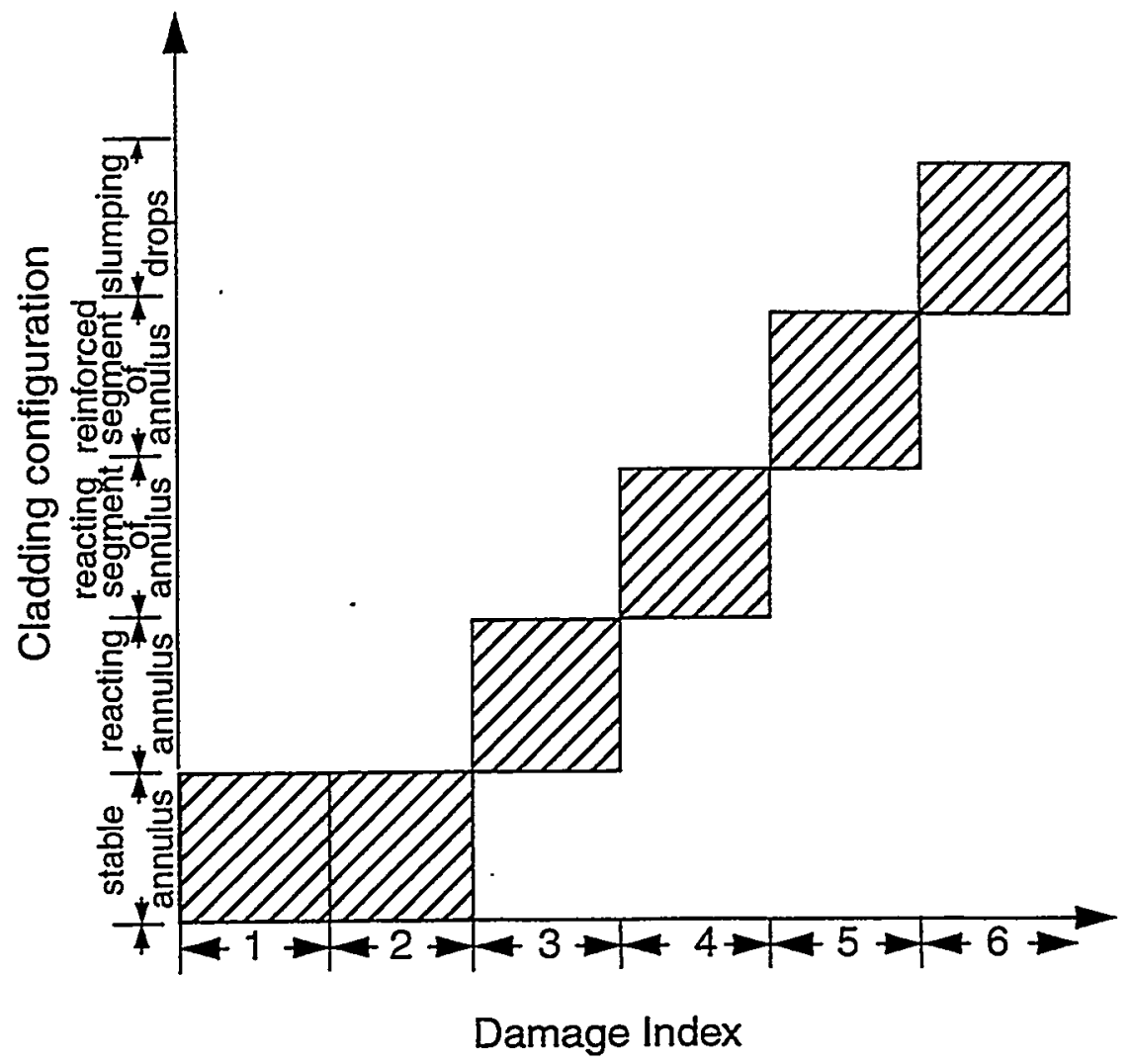

Figure 2. Mapping of cladding configuration as indicated by PIE results.

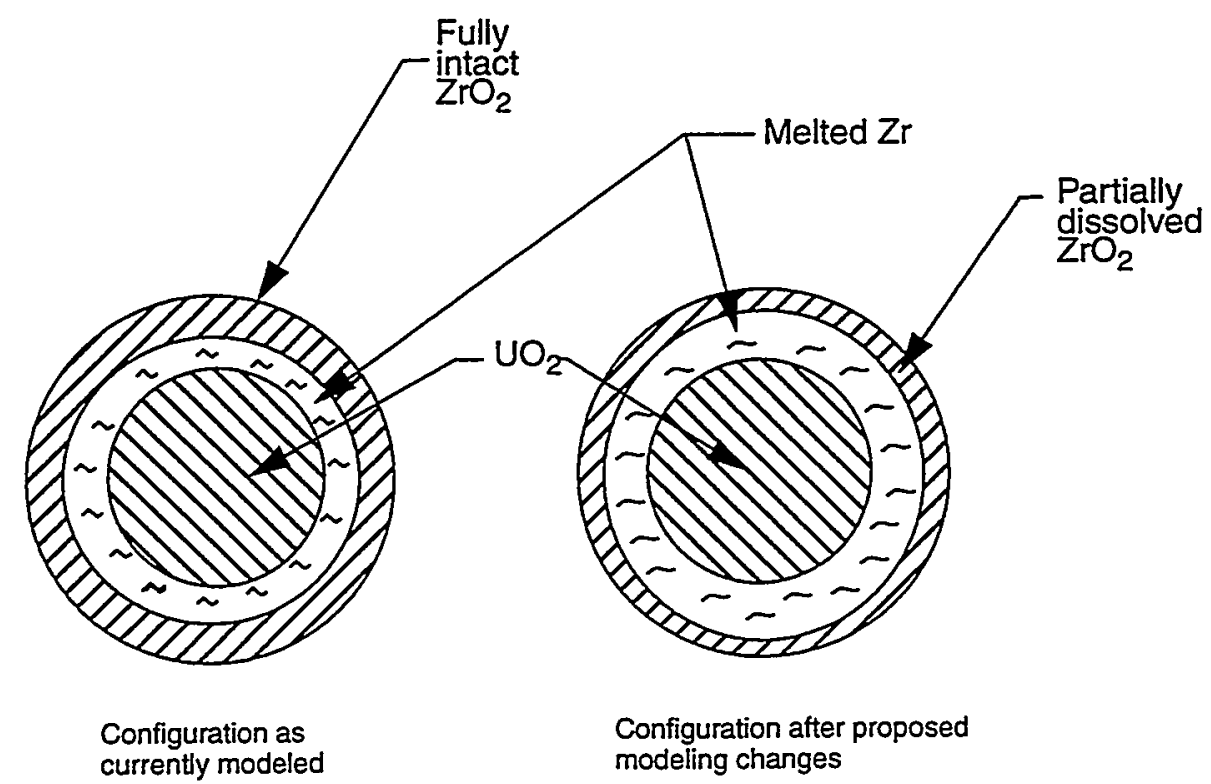

Figure 3. Configuration of cladding for damage index " 3 ". 


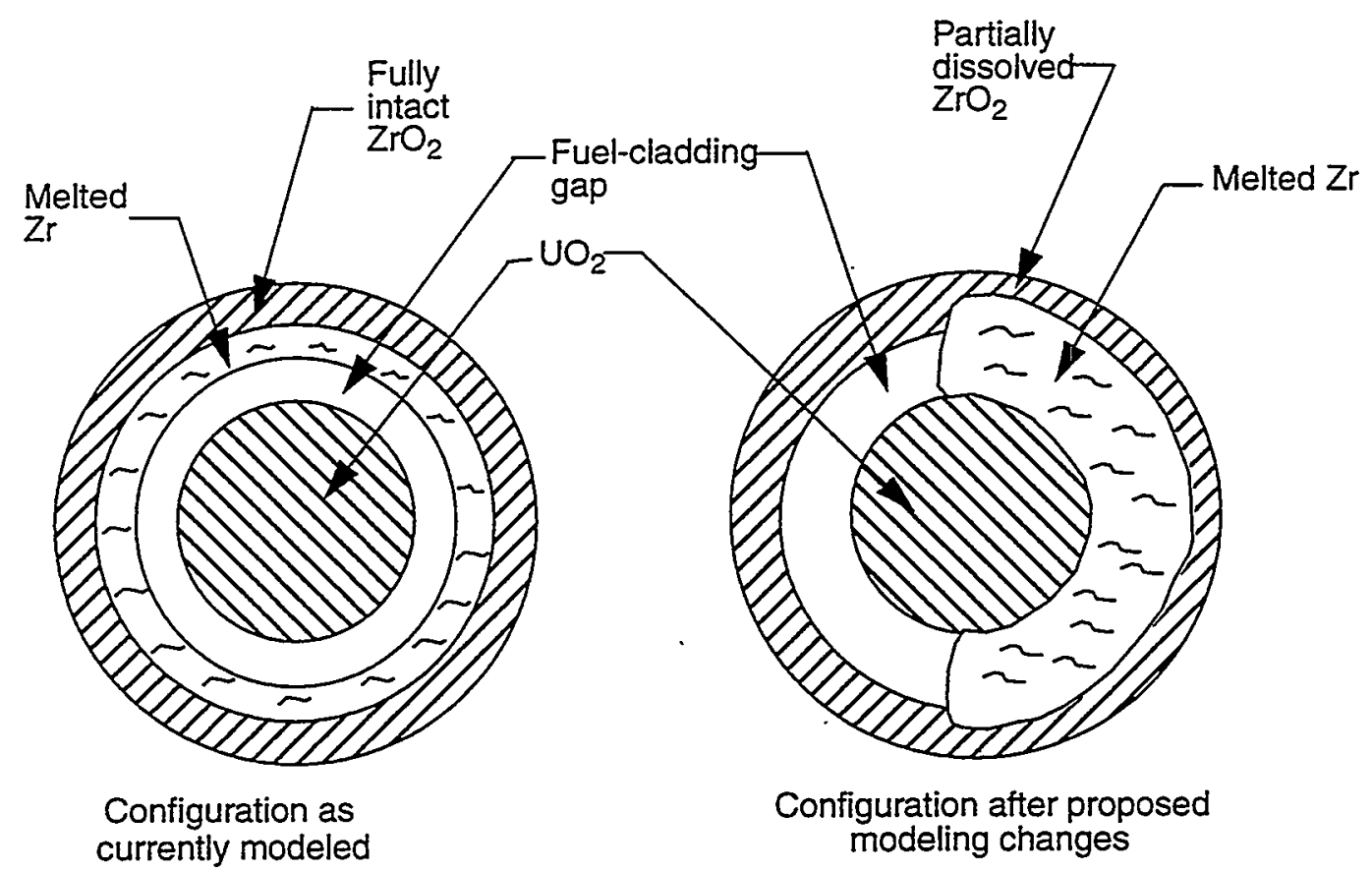

Figure 4. Configuration of cladding for damage index " 4 ".

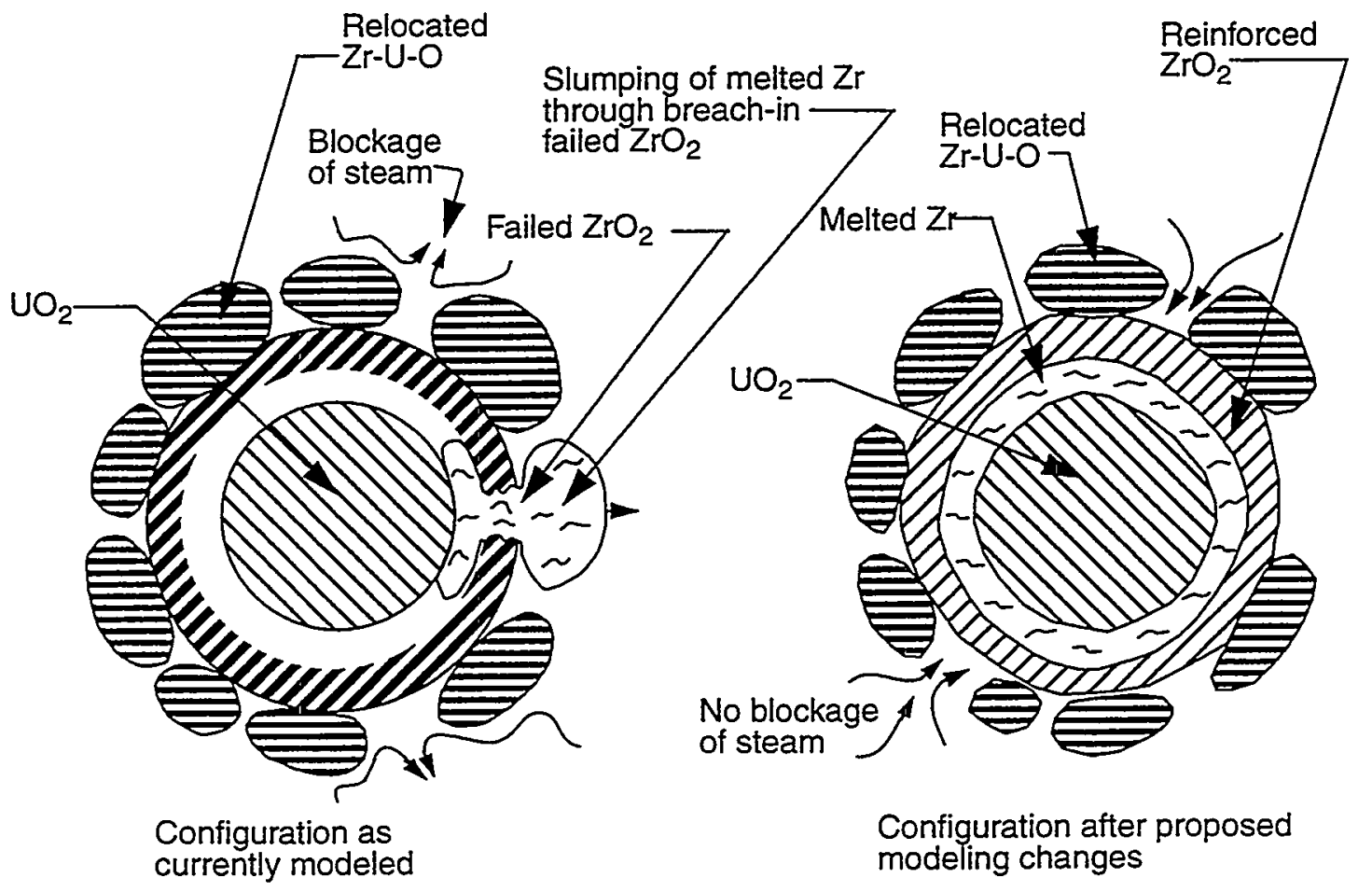

Figure 5. Configuration of cladding for damage index " 5 ". 


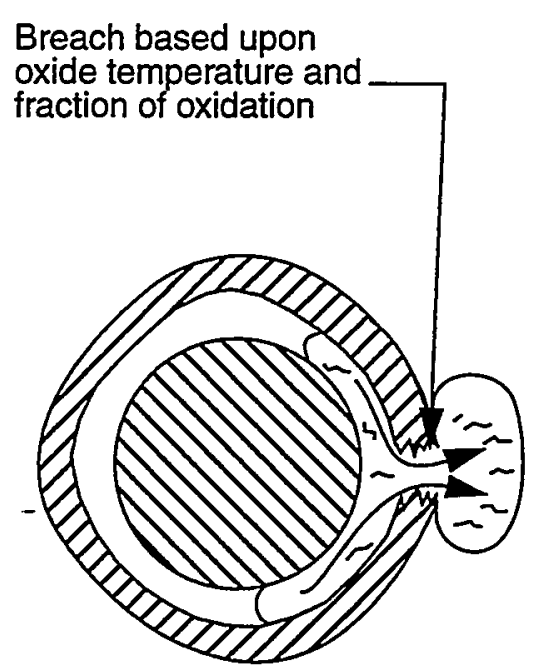

Configuration as currently modeled

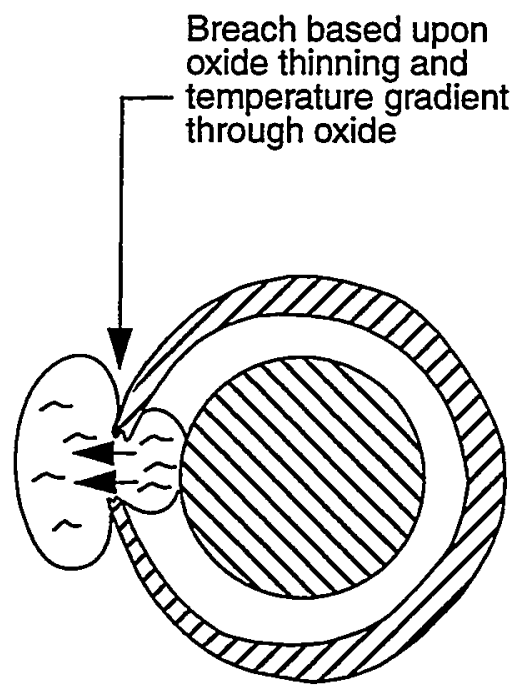

Configuration after proposed modeling changes

Figure 6. Configuration of cladding for damage index "6".

The objectives of this report are two-fold. First, a basis for a correct mapping of the configuration of melted metallic cladding will be established from a compilation of PIE results from severe fuel damage tests. Second, empirical equations will be developed for calculating the integrity of the oxidic part of the cladding as a function of its stress and the extent of its dissolution into the metallic part of the cladding that it contacts. These equations will replace the current criterion for loss of integrity which is based on temperature and extent of oxidation. Each of the proposed modeling changes as indicated in Figure 3 through Figure 6 is expected to contribute to a calculation of less oxidation of the cladding in the upper part of fuel rods and greater oxidation in the lower part of fuel rods. After integration of each of these modeling changes, the calculated axial distribution in cladding oxidation is expected to be in better agreement with experimental results than is currently the case.

The revised cladding configuration mapping and integrity evaluation will be empirical. Mechanistic models for calculating the changes in cladding configuration and integrity as fuel rod heatup and damage progress would require an order of magnitude increase in the spatial detail of calculated cladding behavior. For example, mechanistic modeling to account for the effect of eccentricity of the fuel pellets on fuel cladding chemical reaction would require the introduction of a spatial coordinate in the circumferential direction. As a result, fuel rod behavior would need to be calculated in three dimensions (radial, axial, and circumferential) instead of the current two dimensions (radial, axial). Such detail is beyond the scope of a systems analysis code such as SCDAP/RELAP5. Also, the structural properties of the metallic and oxidic phases of the cladding required for a mechanistic approach are not firmly established at high temperatures (temperatures greater than $2000 \mathrm{~K}$ ). So the best approach to resolving the identified deficiency in 
calculated axial distribution of oxidation is to develop empirical models based on the greatest possible collection of experimental results. These empirical models would be a significant improvement over current modeling that is based on assumptions of cladding configuration mapping that are not consistent with experimental results. Also, the empirical models can be developed and implemented with a significantly less amount of effort than would be required for fully mechanistic models.

The organization of this preliminary design report is as follows. Section 2 presents comparisons of SCDAP/RELAP5/MOD3.2 calculations and experimental results that identify the discrepancy between calculated and measured axial distribution in cladding oxidation. This section also presents observations of cladding relocation. Section 3 presents the PIE results that can be used to establish a basis for the revised mapping of cladding configuration. Section 4 presents the set of rules that will be used to map the configuration of partially oxidized cladding in a manner that is consistent with PIE results. This section also defines the model that will be applied to calculate the integrity of the oxidic part of the cladding that retains the melted metallic part of the cladding. The integration and implementation of the revised models into SCDAP/RELAP5/MOD3.2 is described in Section 5. The plan for testing and assessing the new and improved models is presented in Section 6. A summary of the proposed changes in modeling is presented in Section 7 and the references are presented in Section 8.

\section{Assessment of Calculated Axial Distribution in Oxidation}

This section presents a comparison of calculated and measured axial distributions in cladding oxidation for a range of severe accident experiments. While the overall extent of cladding oxidation was in fairly good agreement with experimental results for these experiments, generally the calculated and measured axial distributions in oxidation were not in good agreement. The general trend was an overprediction of cladding oxidation at the top of the test fuel rods and an underprediction of oxidation at the bottom of fuel rods. In addition, the extent of cladding oxidation generally was significantly underpredicted at locations with a significant amount of relocated material. Five severe accident experiments were identified that had PIE results useful for assessing the calculated and measured axial distributions in cladding oxidation. These five experiments were; (1) PBF SFD1-1 ${ }^{2}$, (2) PBF SFD 1-4 $4^{3}$, (3) CORA- $13^{4}$, (4) PHEBUS B $9+,{ }^{5}$ and OECD LOFT FP- $2^{6}$. The PIE results of these five severe accident experiments from which comparisons can be made of calculated and measured axial distributions in cladding oxidation are presented next. The presented PIE results include the measured variation in the axial direction of the fraction of cladding oxidation and the measured maximum temperature at various elevations in the test bundle. The calculated local temperature at the time that the calculated fraction of oxidation equaled the measured fraction of oxidation is also presented. Other data that may be useful for mapping cladding configuration as a function of damage progression are also presented. This data includes the measured cladding hoop strain as a function of elevation, data indicating whether liquefied cladding was observed to have slumped from a location and whether relocated cladding was observed to have solidified at a location.

The calculated and measured axial distributions in cladding oxidation for the PBF SFD 1-1 test ${ }^{2}$ are compiled in Table 2. The table also includes information on calculated and measured axial temperature profiles and on measured cladding relocation. The maximum calculated temperature at an elevation is indicated by values for extent of oxidation placed in the four columns for temperature; for example, an entry in the " 2300 " column but not in the " 2400 " column indicates that the maximum calculated temperature exceeded $2300 \mathrm{~K}$ but not $2400 \mathrm{~K}$. A column indicating the measured cladding hoop strain is 
placed in the table to indicate the value of this important oxidation parameter. As shown in this table, SCDAP/RELAP5/MOD3.2 significantly underpredicted the extent of cladding oxidation in the elevation interval of $0.1 \mathrm{~m}$ to $0.3 \mathrm{~m}$ and significantly overpredicted the extent of oxidation in the elevation interval of $0.7 \mathrm{~m}$ to $0.8 \mathrm{~m}$. The overprediction in the elevation range of $0.7 \mathrm{~m}$ to $0.8 \mathrm{~m}$ may in part be due to the code not modeling the heatup of cladding caused by its uptake of hydrogen which theoretical analyses have indicated may be of the same order of magnitude as that caused by oxidation ${ }^{8,9}$. Cladding material was measured to slump away from the $0.7 \mathrm{~m}$ to $1.0 \mathrm{~m}$ elevation interval and the $0.3 \mathrm{~m}$ to $0.4 \mathrm{~m}$ elevation interval and to slump to the $0.05 \mathrm{~m}$ to $0.2 \mathrm{~m}$ elevation interval. The maximum concentration of relocated material occurred in the elevation interval of $0.05 \mathrm{~m}$ to $0.20 \mathrm{~m}$, wherein all the space between the fuel rods in the center part of the test bundle was filled with relocated material. As shown in the table, SCDAP/ RELAP5/MOD3.2 significantly underpredicted the temperature at the elevation of $0.14 \mathrm{~m}$, where all of the space between the fuel rods was filled with relocated material. At this elevation, the maximum temperature was calculated to be less than $2300 \mathrm{~K}$ (indicated by no number entered in the $2300 \mathrm{~K}$ column of table), but the PIE results showed that the maximum temperature was $2890 \mathrm{~K}$. The hot temperature shown by the PIE results and the-100\% oxidation of the fuel rod cladding at this elevation indicate that the relocated material did not completely block oxidation at this location.

Table 2. Comparison of calculated and measured axial distribution in cladding oxidation for PBF SFD 11 test

\begin{tabular}{|c|c|c|c|c|c|c|c|c|}
\hline \multirow{2}{*}{$\frac{\text { Elevation }}{\text { (m) }}$} & \multirow{2}{*}{$\begin{array}{c}\text { PIE } \\
\text { indicated } \\
\begin{array}{c}\text { maximum } \\
\text { temp. }\end{array} \\
(\mathrm{K})\end{array}$} & \multicolumn{4}{|c|}{$\begin{array}{l}\text { Calculated fraction of cladding } \\
\text { oxidized when calculated } \\
\text { temperature }(\mathrm{K})>\end{array}$} & \multirow{2}{*}{$\begin{array}{l}\text { measured } \\
\text { slumping? } \\
\text { /measured } \\
\text { blockage? }\end{array}$} & \multirow{2}{*}{$\begin{array}{l}\text { Measured/ } \\
\text { calculated } \\
\text { fraction of } \\
\text { cladding } \\
\text { oxidized }\end{array}$} & \multirow{2}{*}{$\begin{array}{l}\text { measured } \\
\text { hoop } \\
\text { strain }\end{array}$} \\
\hline & & 2300 & 2400 & 2500 & 2600 & & & \\
\hline 0.04 & 2200 & & & & & no/no & $0.60 / 0.0$ & $<0.01$ \\
\hline 0.14 & 2890 & & & & & notyes & $1.00 / 0.51$ & $<0.01$ \\
\hline 0.23 & 2560 & 0.13 & & & & no/no & $1.00 / 0.13$ & 0.20 \\
\hline 0.33 & 2240 & 0.16 & 0.16 & 0.16 & 0.16 & yes/no & $0.25 / 0.16$ & 0.32 \\
\hline 0.46 & 2180 & 0.24 & 0.29 & 0.36 & 0.36 & no/no & $0.65 / 0.36$ & 0.30 \\
\hline 0.61 & 2190 & 0.21 & 0.25 & 0.38 & 0.38 & no/no & $0.45 / 0.38$ & 0.30 \\
\hline 0.74 & 2260 & 0.22 & 0.39 & 0.42 & 0.46 & yes/no & $0.11 / 0.46$ & 0.30 \\
\hline 0.88 & $>1860$ & 0.28 & 0.30 & 0.32 & 0.34 & yes/no & $0.20 / 0.34$ & 0.10 \\
\hline
\end{tabular}

The calculated and measured axial distributions in cladding oxidation for the PBF SFD 1-4 test ${ }^{3}$ are compiled in Table 3. As shown in this table, SCDAP/RELAP5/MOD3.2 significantly underpredicted the extent of cladding oxidation in the elevation interval of $0.1 \mathrm{~m}$ to $0.3 \mathrm{~m}$ and significantly overpredicted the extent of oxidation in the elevation interval of $0.8 \mathrm{~m}$ to $1.0 \mathrm{~m}$. The overprediction in the elevation range of 
$0.8 \mathrm{~m}$ to $1.0 \mathrm{~m}$ may in part be due to the code not modeling the dissolution of $\mathrm{ZrO}_{2}$ by melted $\mathrm{Zr}$ and in part due to the code not modeling the heatup of cladding caused by its uptake of hydrogen. Cladding was measured to slump away from the $0.30 \mathrm{~m}$ to $1.0 \mathrm{~m}$ elevation interval and to slump to the $0.20 \mathrm{~m}$ to $0.40 \mathrm{~m}$ elevation interval. The maximum concentration of relocated material occurred in the elevation interval of $0.20 \mathrm{~m}$ to $0.30 \mathrm{~m}$, wherein all the space between the fuel rods in the center part of the test bundle was filled with relocated material. A significant concentration of relocated metallic material also occurred in the elevation interval of $0.05 \mathrm{~m}$ to 0.20 $\mathrm{m}$. Some ceramic and metallic material relocated into the elevation interval of 0.30 to $0.40 \mathrm{~m}$. The comparisons of maximum calculated temperature and maximum PIE indicated temperature indicate that the code probably overpredicted the temperature in the elevation interval of $0.7 \mathrm{~m}$ to $0.8 \mathrm{~m}$.

The PIE results indicated that a large concentration of relocated material may not slump until its temperature exceeds $2800 \mathrm{~K}$. In the elevation interval of $0.15 \mathrm{~m}$ to $0.30 \mathrm{~m}$, wherein a large concentration of relocated material was present, the PIE results showed that material at a temperature of about $2800 \mathrm{~K}$ slumped into this elevation interval. The in-place material in this elevation interval did not exceed a temperature of 2400 $\mathrm{K}$. Two scenarios for material relocation are consistent with the PIE results. One scenario is that a large concentration of previously relocated material at a temperature of $2800 \mathrm{~K}$ slumped into this elevation interval. The other scenario is that an the oxide layer above the $0.3 \mathrm{~m}$ elevation that was retaining liquefied material did not fail until its temperature exceeded its melting temperature, which is about $2800 \mathrm{~K}$.

Table 3. Comparison of calculated and measured axial distributions in extent of cladding oxidation for PBF SFD $1-4$ test

\begin{tabular}{|c|c|c|c|c|c|c|c|c|}
\hline \multirow{2}{*}{$\frac{\text { Elevation }}{\text { (m) }}$} & \multirow{2}{*}{$\begin{array}{c}\text { PIE } \\
\text { indicated } \\
\text { maximum } \\
\text { temp. }\end{array}$} & \multicolumn{4}{|c|}{$\begin{array}{c}\text { Calculated fraction of cladding } \\
\text { oxidized when calculated } \\
\text { temperature }(\mathrm{K})>\end{array}$} & \multirow{2}{*}{$\begin{array}{l}\text { Measured } \\
\text { slumping? } \\
\text { /measured } \\
\text { blockage? }\end{array}$} & \multirow{2}{*}{$\begin{array}{c}\text { Measured/ } \\
\text { calculated } \\
\text { fraction of } \\
\text { cladding } \\
\text { oxidized }\end{array}$} & \multirow{2}{*}{$\begin{array}{l}\text { Measured } \\
\text { hoop } \\
\text { strain }\end{array}$} \\
\hline & & 2300 & 2400 & 2500 & 2600 & & & \\
\hline 0.05 & $>1200$ & & & & & notyes & $0.10 / 0.0$ & $<0.01$ \\
\hline 0.15 & $>2000$ & & & & & nolyes & $1.00 / 0.02$ & $<0.01$ \\
\hline 0.25 & $>2200$ & 0.11 & 0.11 & 0.11 & & nolyes & $1.00 / 0.11$ & $<0.05$ \\
\hline 0.35 & $>2600$ & 0.35 & 0.40 & 0.52 & 0.52 & yes/yes & $0.50 / 0.52$ & $0.20 ?$ \\
\hline 0.55 & $>2800$ & 0.28 & 0.33 & 0.37 & 0.40 & yes/no & $<0.30 / 0.37$ & $0.30 ?$ \\
\hline 0.75 & $>2200$ & 0.21 & 0.24 & 0.26 & 0.26 & yes/no & $0.37 / 0.26$ & $0.30 ?$ \\
\hline 0.85 & $>2200$ & 0.29 & 0.50 & & & yes/no & $0.22 / 0.86$ & $0.30 ?$ \\
\hline
\end{tabular}

The calculated and measured axial distributions in cladding oxidation for the KfK CORA-13 experiment ${ }^{4}$ are compiled in Table 4. Entries in the table with a question mark indicate the experimental results did not evaluate that entry or the measured value has a large uncertainty. This experiment reflooded a partially oxidized hot test bundle. As shown in this table, SCDAP/RELAP5/MOD3.2 significantly underpredicted the extent of cladding oxidation in the elevation interval of $0.4 \mathrm{~m}$ to $0.5 \mathrm{~m}$, wherein the maximum blockage occurred, and 
significantly overpredicted the extent of oxidation in the elevation interval of $0.8 \mathrm{~m}$ to $1.0 \mathrm{~m}$. The overprediction of oxidation in the elevation range of $0.8 \mathrm{~m}$ to $1.0 \mathrm{~m}$ may in part be due to the code not modeling the dissolution of $\mathrm{ZrO}_{2}$ by liquefied $\mathrm{Zr}$ and in part due to the code not modeling the heatup of cladding caused by its uptake of hydrogen. Cladding was measured to slump away from the $0.5 \mathrm{~m}$ to $1.0 \mathrm{~m}$ elevation interval and to slump to the $0.4 \mathrm{~m}$ to $0.5 \mathrm{~m}$ elevation interval. The maximum concentration of relocated material occurred in the elevation interval of $0.40 \mathrm{~m}$ to $0.50 \mathrm{~m}$, wherein all the space between the fuel rods in the center part of the test bundle was filled with relocated material. A significant concentration of relocated control rod material occurred in the elevation interval of $0.15 \mathrm{~m}$ to $0.35 \mathrm{~m}$. The comparisons of maximum calculated temperatures and maximum PIE indicated temperatures indicate that the code overpredicted the maximum temperature at all elevations except the $0.6 \mathrm{~m}$ to $0.8 \mathrm{~m}$ elevation interval. The degradation of the test bundle precluded identification the region of the test bundle where double-sided oxidation occurred.

Table 4. Comparison of calculated and measured axial distributions in extent of cladding oxidation for KfK CORA-13 test.

\begin{tabular}{|c|c|c|c|c|c|c|c|c|}
\hline \multirow{2}{*}{ Elevation } & \multirow{2}{*}{$\begin{array}{c}\begin{array}{c}\text { Measured } \\
\text { maximum } \\
\text { temp. }\end{array} \\
(\mathrm{K})\end{array}$} & \multicolumn{4}{|c|}{$\begin{array}{c}\text { Calculated fraction of cladding } \\
\text { oxidized when calculated } \\
\text { temperature }(\mathrm{K})>\end{array}$} & \multirow{2}{*}{$\begin{array}{l}\text { Measured } \\
\text { slumping? } \\
\text { /measured } \\
\text { blockage? }\end{array}$} & \multirow{2}{*}{$\begin{array}{l}\text { Fraction } \\
\text { cladding } \\
\text { oxidized: } \\
\text { measured/ } \\
\text { calculated }\end{array}$} & \multirow{2}{*}{$\begin{array}{l}\text { Measured } \\
\text { hoop } \\
\text { strain }\end{array}$} \\
\hline & & 2300 & 2400 & 2500 & 2600 & & & \\
\hline 0.05 & $<1273$ & 0.17 & 0.19 & 0.26 & & no/no & $0.0 / 0.21$ & $<0.10$ \\
\hline 0.15 & 1273 & 0.15 & 0.18 & 0.18 & 0.18 & no/no & $0.0 / 0.17$ & $<0.10$ \\
\hline 0.25 & 1530 & 0.36 & 0.45 & 0.46 & & nóno & $0.07 / 0.43$ & $<0.10$ \\
\hline 0.35 & 1880 & 0.69 & 0.69 & 0.69 & 0.69 & no/no & $0.57 / 0.70$ & $<0.10$ \\
\hline 0.45 & 1970 & 0.08 & 0.08 & 0.11 & 0.11 & nolyes & $1.0 / 0.10$ & $<0.10$ \\
\hline 0.55 & 2130 & 0.08 & 0.08 & 0.11 & 0.14 & yes/no & $<1.0 / 0.16$ & 0.30 \\
\hline 0.65 & $?$ & 0.25 & 0.29 & 0.36 & 0.41 & yes/no & $<1.0 / 0.36$ & 0.30 \\
\hline 0.75 & $?$ & 0.07 & 0.07 & 0.11 & 0.11 & yes/no & $<1.0 / 0.20$ & 0.30 \\
\hline 0.85 & 2130 & 0.20 & 0.22 & 0.24 & & yes/no & $<0.05 / 0.43$ & 0.30 \\
\hline 0.95 & 2130 & 0.18 & 0.21 & 0.23 & 0.23 & yes/no & $<0.05 / 0.40$ & 0.30 \\
\hline
\end{tabular}

The calculated and measured axial distributions in cladding oxidation for the PHEBUS B9+ experiment ${ }^{5}$ are shown in Table 5. This test provides information on cladding behavior for the case of extreme steam starvation. In this experiment, the steam flow through the bundle was replaced with helium flow after the bundle had increased in temperature to the point where rapid oxidation of fuel rod cladding was occurring. The PIE results available for this experiment are considerably less than those available from the previous three experiments. Therefore, all of the values except the measured fraction of cladding oxidation are calculated values. The table shows that a significant discrepancy between the calculated and 
measured extent of cladding oxidation occurred at the 0.44 m elevation, where the cladding was calculated to be completely oxidized but the measurement indicated that the cladding was only $40 \%$ oxidized. This discrepancy is considered to be due to the calculation of double-sided oxidation at this location where in reality the oxidation was single-sided. The PIE results indicated that the maximum blockage occurred at the elevation of $0.26 \mathrm{~m}$, where all of the space between the fuel rods was filled with relocated material in part of the bundle cross section. The temperatures in the bottom $0.4 \mathrm{~m}$ of the test bundle were calculated to become very hot (temperature $>2500 \mathrm{~K}$ ) only during the helium flow phase of the experiment. As a result, the calculated fractions of cladding oxidation in this elevation interval are relatively small considering the temperatures attained in this elevation interval.

Table 5. Comparison of calculated and measured axial distributions in extent of cladding oxidation for PHEBUS B9+ test.

\begin{tabular}{|c|c|c|c|c|c|c|c|c|}
\hline \multirow{2}{*}{ Elevation } & \multirow{2}{*}{$\begin{array}{c}\begin{array}{c}\text { Maximum } \\
\text { calculated } \\
\text { temp. }\end{array} \\
(\mathrm{K})\end{array}$} & \multicolumn{4}{|c|}{$\begin{array}{l}\text { Calculated fraction of outer half } \\
\text { of cladding oxidized when } \\
\text { calculated temperature }(\mathrm{K})>\end{array}$} & \multirow{2}{*}{$\begin{array}{l}\text { Calculated } \\
\text { slumping? } \\
\text { /blockage? }\end{array}$} & \multirow{2}{*}{$\begin{array}{l}\text { Fraction } \\
\text { cladding } \\
\text { oxidized: } \\
\text { measured/ } \\
\text { calculated }\end{array}$} & \multirow{2}{*}{$\begin{array}{l}\text { Calculated } \\
\text { hoop } \\
\text { strain }\end{array}$} \\
\hline & & 2300 & 2400 & 2500 & 2600 & & & \\
\hline 0.04 & 2691 & 0.03 & 0.03 & 0.03 & 0.03 & yes/no & $0.0 / 0.02$ & 0.01 \\
\hline 0.12 & 2831 & 0.07 & 0.07 & 0.07 & 0.07 & yes/yes & $0.03 / 0.06$ & 0.01 \\
\hline 0.20 & 2867 & 0.13 & 0.13 & 0.13 & 0.13 & yes/no & $0.05 / 0.11$ & 0.01 \\
\hline 0.28 & 2579 & 0.22 & 0.22 & 0.22 & & yes/yes & $0.10 / 0.21$ & 0.02 \\
\hline 0.36 & 2565 & 0.33 & 0.33 & 0.33 & 0.33 & yes/no & $0.25 / 0.31$ & 0.02 \\
\hline 0.44 & 2580 & 0.43 & 0.43 & 0.43 & & yes/no & $0.40 / 0.86$ & 0.08 \\
\hline 0.52 & 2698 & 1.00 & 1.0 & 1.0 & 1.0 & yes/no & $0.70 / 1.0$ & 0.15 \\
\hline 0.60 & 2666 & 1.0 & 1.0 & 1.0 & 1.0 & yes/no & $1.0 / 1.0$ & 0.20 \\
\hline 0.68 & 2541 & 1.0 & 1.0 & 1.0 & & no/no & $1.0 / 1.0$ & 0.13 \\
\hline 0.76 & 2139 & 0.70 & 0.70 & 0.70 & 0.70 & no/no & $0.75 / 0.52$ & 0.02 \\
\hline
\end{tabular}

The calculated and measured axial distributions in cladding oxidation for the OECD LOFT FP-2 experiment ${ }^{6}$ are shown in Table 6 . This test provides information on the behavior of fuel rod cladding for cases in which reflood occurs and the maximum fuel bundle temperature exceeds $3000 \mathrm{~K}$. This table also presents the SCDAP/RELAP5/MOD3.2 calculations of the extent of cladding oxidation as a function of the calculated temperature exceeding a range of thresholds. 
The results in Table 6 indicate that SCDAP/RELAP5/MOD3.2 significantly overpredicted the extent

Table 6. Comparison of calculated and measured axial distributions in extent of cladding oxidation for OECD LOFT FP-2 test.

\begin{tabular}{|c|c|c|c|c|c|c|c|c|}
\hline \multirow{2}{*}{$\begin{array}{l}\text { Elevation } \\
\text { (m) }\end{array}$} & \multirow{2}{*}{ 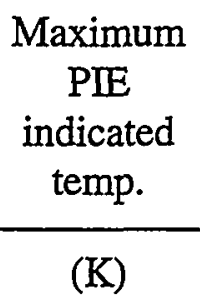 } & \multicolumn{4}{|c|}{$\begin{array}{l}\text { Calculated fraction of outer half } \\
\text { of cladding oxidized when } \\
\text { calculated temperature }(K)>\end{array}$} & \multirow{2}{*}{$\begin{array}{l}\text { Measured } \\
\text { slumping? } \\
\text { /measured } \\
\text { blockage? }\end{array}$} & \multirow{2}{*}{$\begin{array}{l}\text { Fraction } \\
\text { cladding } \\
\text { oxidized: } \\
\text { measured/ } \\
\text { calculated }\end{array}$} & \multirow{2}{*}{$\begin{array}{l}\text { Measured } \\
\text { hoop } \\
\text { strain }\end{array}$} \\
\hline & & 2300 & 2400 & 2500 & 2600 & & & \\
\hline 0.48 & 1800 & & & & & notyes & $0.02 / 0.90$ & 0.05 \\
\hline 0.66 & $>2880$ & 0.90 & & & & nolyes & $>0.26 / 0.90$ & $<0.30$ \\
\hline 0.77 & $>3120$ & 0.80 & 0.80 & 0.80 & 0.13 & nolyes & $1.0 / 0.80$ & 0.30 \\
\hline 0.88 & $>2960$ & 0.35 & 0.40 & 0.52 & & nolyes & $1.0 / 0.76$ & 0.30 \\
\hline 1.04 & $>2530$ & 0.76 & & & & no/no & $1.0 / 0.76$ & 0.30 \\
\hline 1.20 & $>2330$ & & & & & yes/no & $0.69 / 0.77$ & 0.30 \\
\hline 1.30 & $>2220$ & & & & & yes/no & $<0.5 ? / 0.83$ & $<0.30 ?$ \\
\hline 1.45 & $>2080$ & & & & & yes/no & $<0.5 ? / 0.68$ & $<0.30 ?$ \\
\hline 1.68 & $>1820$ & & & & & yes/no & $<0.5 ? / 0.91$ & $<0.30 ?$ \\
\hline
\end{tabular}

. of cladding oxidation in the elevation interval of $1.30 \mathrm{~m}$ to $1.68 \mathrm{~m}$ and also overpredicted the extent of oxidation at the $0.48 \mathrm{~m}$ elevation. The overprediction of oxidation in the elevation range of $1.30 \mathrm{~m}$ to 1.68 $m$ may in part be due to the code not modeling the dissolution of $\mathrm{ZrO}_{2}$ by $\mathrm{Zr}$ and in part due to the code not modeling the heatup of cladding caused by its uptake of hydrogen. The overprediction of the cladding oxidation at the $0.48 \mathrm{~m}$ elevation is due to an overprediction of the temperature at this elevation; the maximum temperature was calculated to be $2250 \mathrm{~K}$ but the maximum temperature as indicated by the PIE results was only $1800 \mathrm{~K}$.

For the OECD LOFT FP-2 experiment, cladding was observed to slump away from the $1.1 \mathrm{~m}$ to 1.68 $\mathrm{m}$ elevation interval and to slump to the $0.5 \mathrm{~m}$ to $0.9 \mathrm{~m}$ elevation interval. The maximum blockage from relocated material occurred in the elevation interval of $0.66 \mathrm{~m}$ to $0.77 \mathrm{~m}$, where all of the space between the fuel rods in part of the fuel bundle was filled with relocated material that was completely oxidized. In the elevation interval of $0.8 \mathrm{~m}$ to $1.4 \mathrm{~m}$, only small concentrations of relocated material were found. All of the relocated material in this elevation interval was completely oxidized. The PIE results showed that there were no metallic layers of cladding in the elevation interval of $0.66 \mathrm{~m}$ to $1.68 \mathrm{~m}$ (top of active fuel stack) and the layers of oxidized cladding in this elevation interval were considerably thinner than the thickness completely oxidized cladding. As a result, it can be inferred for this elevation interval that the cladding oxide lost its structural integrity and released the liquefied metallic cladding it was retaining or that some of the metallic cladding reacted with the fuel. Some removal of fuel from the outer part of the fuel pellet stack should be evident at locations where liquefied cladding with dissolved fuel flowed away. Since there 
is no evidence of this removal in the elevation span of $0.66 \mathrm{~m}$ to $1.04 \mathrm{~m}$ (except at the elevation of $0.88 \mathrm{~m}$ ), it can be inferred that in this elevation interval the oxide layer for the most part retained liquefied material. On the other hand, at the elevation of $0.77 \mathrm{~m}$ the oxide layer was calculated to lose its structural integrity and release the liquefied material it had retained. This discrepancy between PIE results and calculations is apparently due to deficiencies in modeling the effect of relocated material on the structural integrity of the oxide layer. In the elevation interval of $1.04 \mathrm{~m}$ to $1.68 \mathrm{~m}$, the PIE results indicated that cladding meltdown occurred but cladding meltdown was calculated not to occur in this elevation interval. This discrepancy between calculations and PIE results was not necessarily due to discrepancies in modeling the structural integrity the cladding oxide layer; the maximum temperature calculated for this elevation interval was less than the melting temperature of the metallic part of the cladding. This discrepancy in temperature was in part due to the lack of a model to calculate the heatup due to hydrogen uptake and in part due to an underprediction of cladding ballooning in this elevation interval.

The PIE results indicated that cladding ballooning to the point of rod to rod contact occurred in the elevation interval of $0.77 \mathrm{~m}$ to $1.20 \mathrm{~m}$. Ballooning to the point of rod-to-rod contact corresponds with a cladding hoop strain of about 0.30 . The large extent of bundle degradation in the elevation interval of large cladding hoop strains precluded the PIE results from identifying whether the cladding oxidation in this region was single-sided or double-sided.

\section{PIE Results for Mapping Cladding Configuration}

This section presents PIE results that can be used as a basis for mapping the configuration of fuel rod cladding as a function of damage progression. The PIE results were obtained from the five severe fuel damage experiments referenced in the previous section, namely the PBF SFD 1-1 test, ${ }^{2}$ PBF SFD $1-4$ test, $^{3}$ KfK CORA-13 Test, ${ }^{4}$ PHEBUS B9+ test, ${ }^{5}$ and the OECD LOFT FP-2 test ${ }^{6}$.

At locations where cladding ballooning and melting has occurred, PIE results indicate that the liquefied metallic layer generally has an asymmetric configuration. Figure 7 shows an example of the diverse possibilities for the configuration of the liquefied material layer for two adjacent fuel rods. ${ }^{6}$ In one fuel rod, the configuration of the metallic layer is almost annular in shape and in an adjacent rod the metallic layer has significant asymmetry. Further examples from PIE results for the OECD LOFT FP-2 test of an asymmetric configuration of a liquefied metallic layer are shown in Figure 8 through Figure 11. An example of an asymmetric shape of liquefied cladding and dissolved fuel from the PIE results of the PBF SFD 1-1 experiment ${ }^{2}$ is shown in Figure 12 . No PIE results were found that indicate a metallic layer stays sandwiched between the inner and outer oxide layers after the metallic layer has liquefied. In some cases the metallic part of the cladding is concentrated in a 90 degree circumferential segment of the cladding and in other cases the metallic part is spread over most of the circumference. The average value of the circumferential segment retaining liquefied metallic cladding is estimated to be about 225 degrees. 


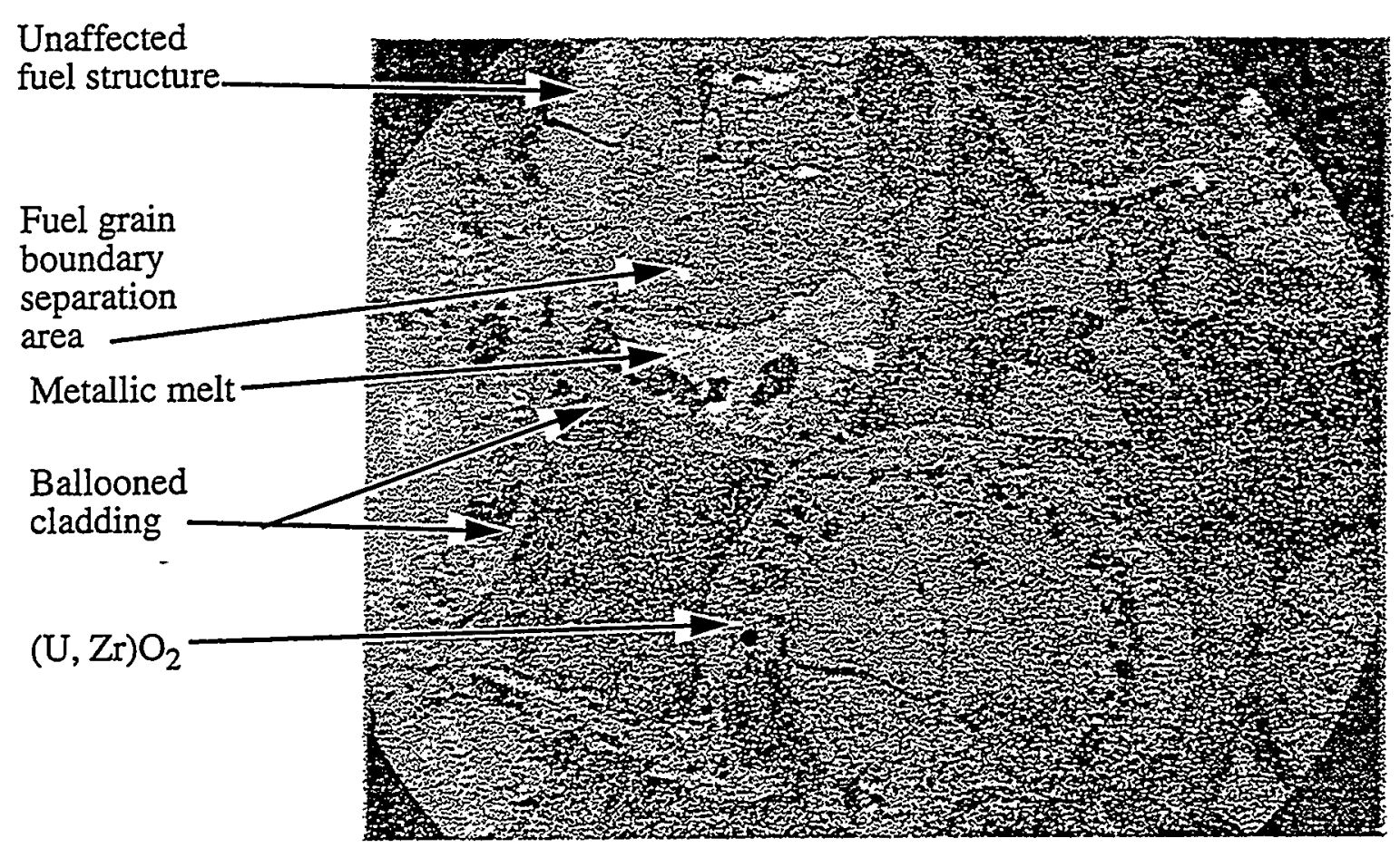

Figure 7. Example of diverse configurations of liquefied metallic layer from PIE results of OECD LOFT FP-2 experiment.

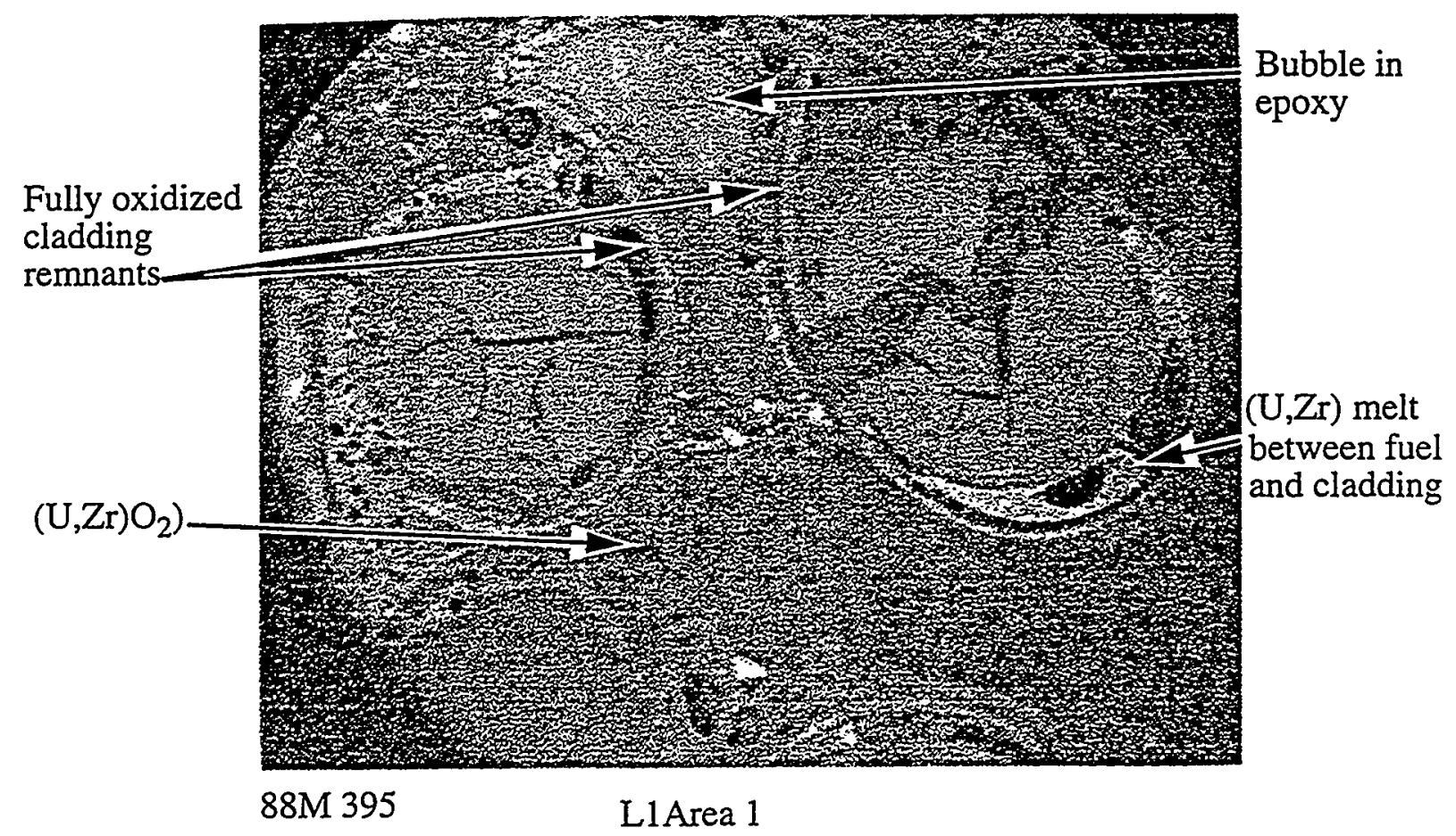

Figure 8. Example of asymmetric configuration of liquefied metallic layer from $0.96 \mathrm{~m}$ elevation of OECD LOFT FP-2 Test bundle (L1 Area 1). 


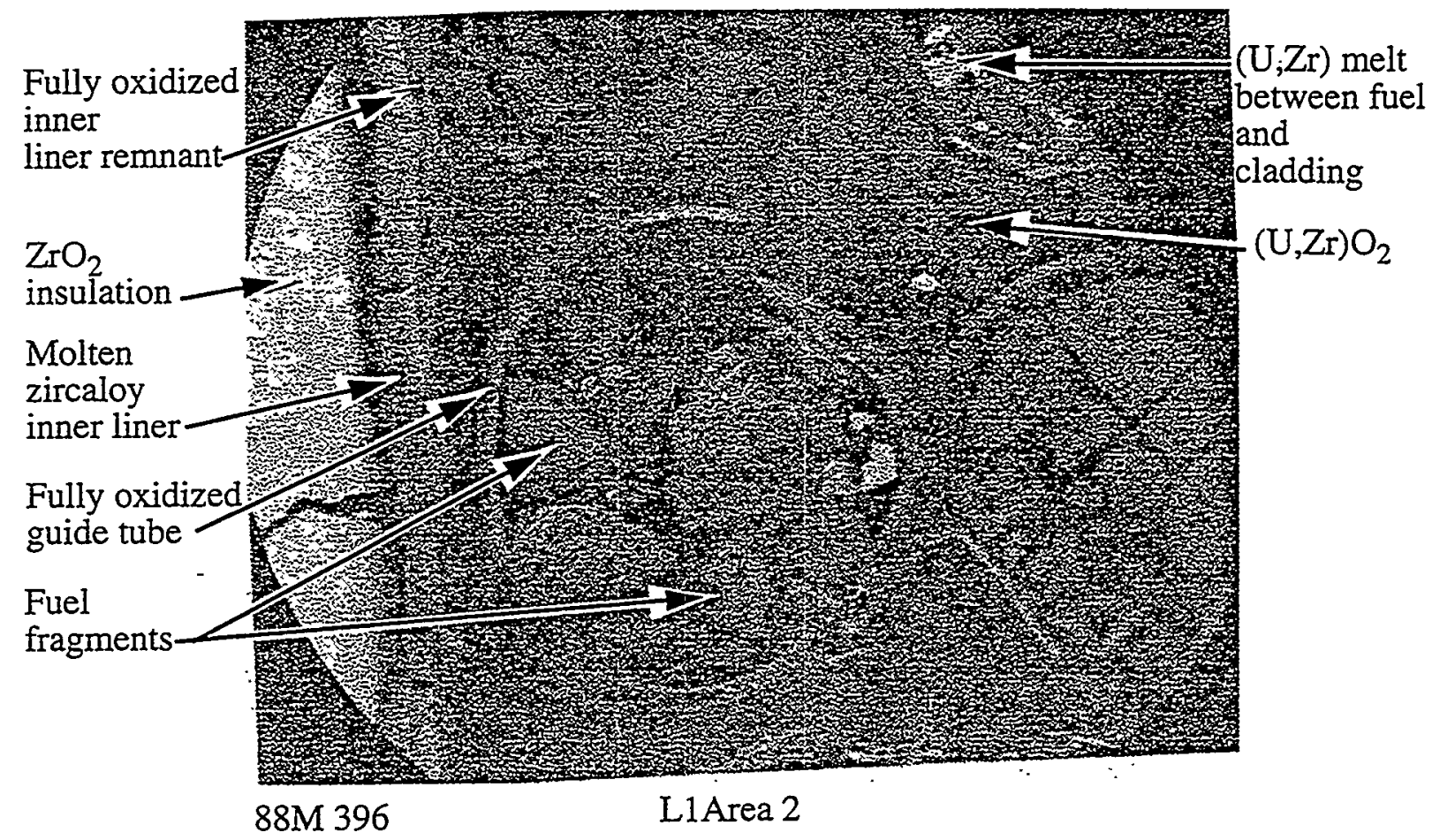

Figure 9. Example of asymmetric configuration of liquefied metallic layer from $0.96 \mathrm{~m}$ elevation of OECD LOFT FP-2 Test bundle (L1Area2).

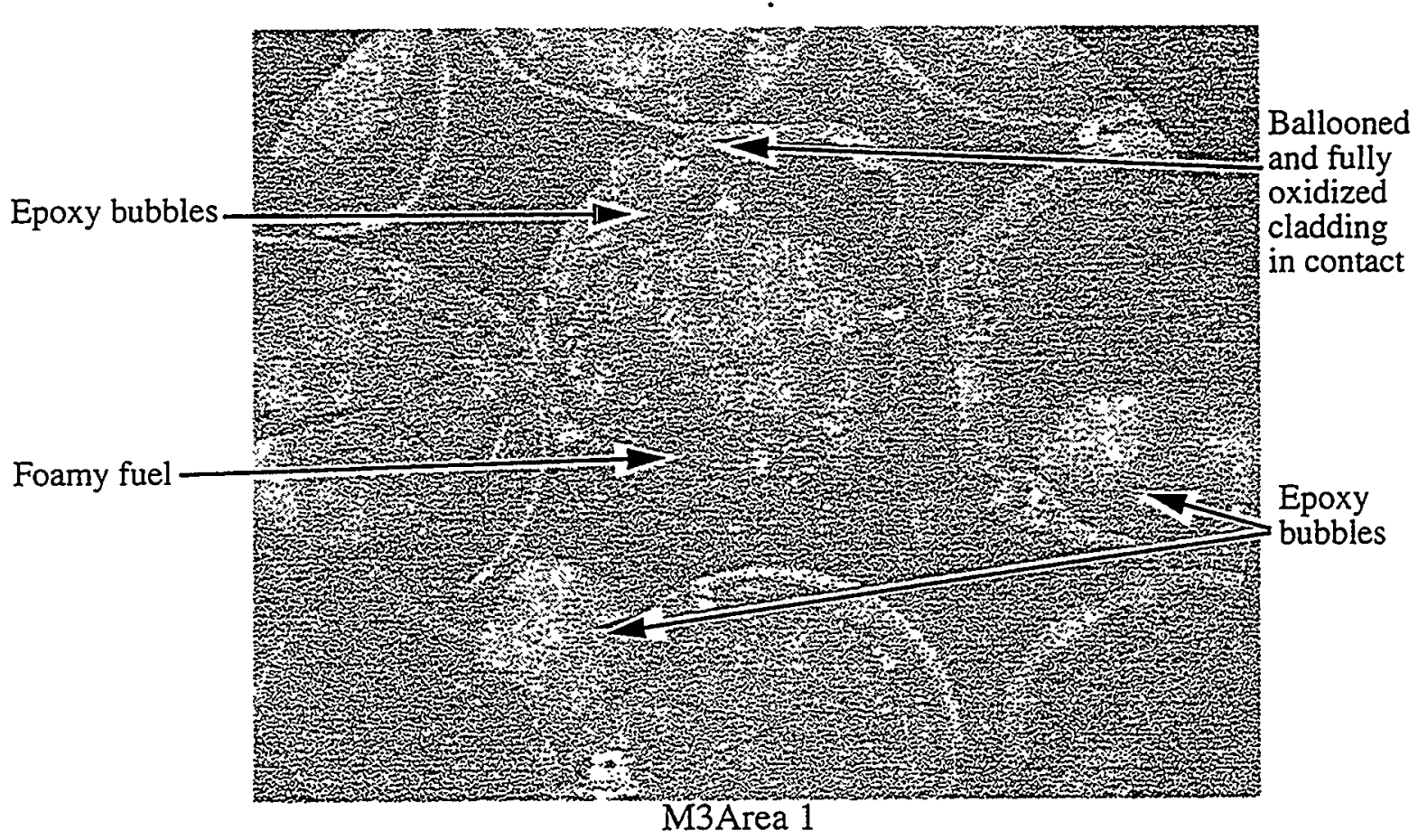

Figure 10. Example of asymmetric configuration of liquefied metallic layer from $1.04 \mathrm{~m}$ elevation of OECD LOFT FP-2 test bundle (M3Area1). 


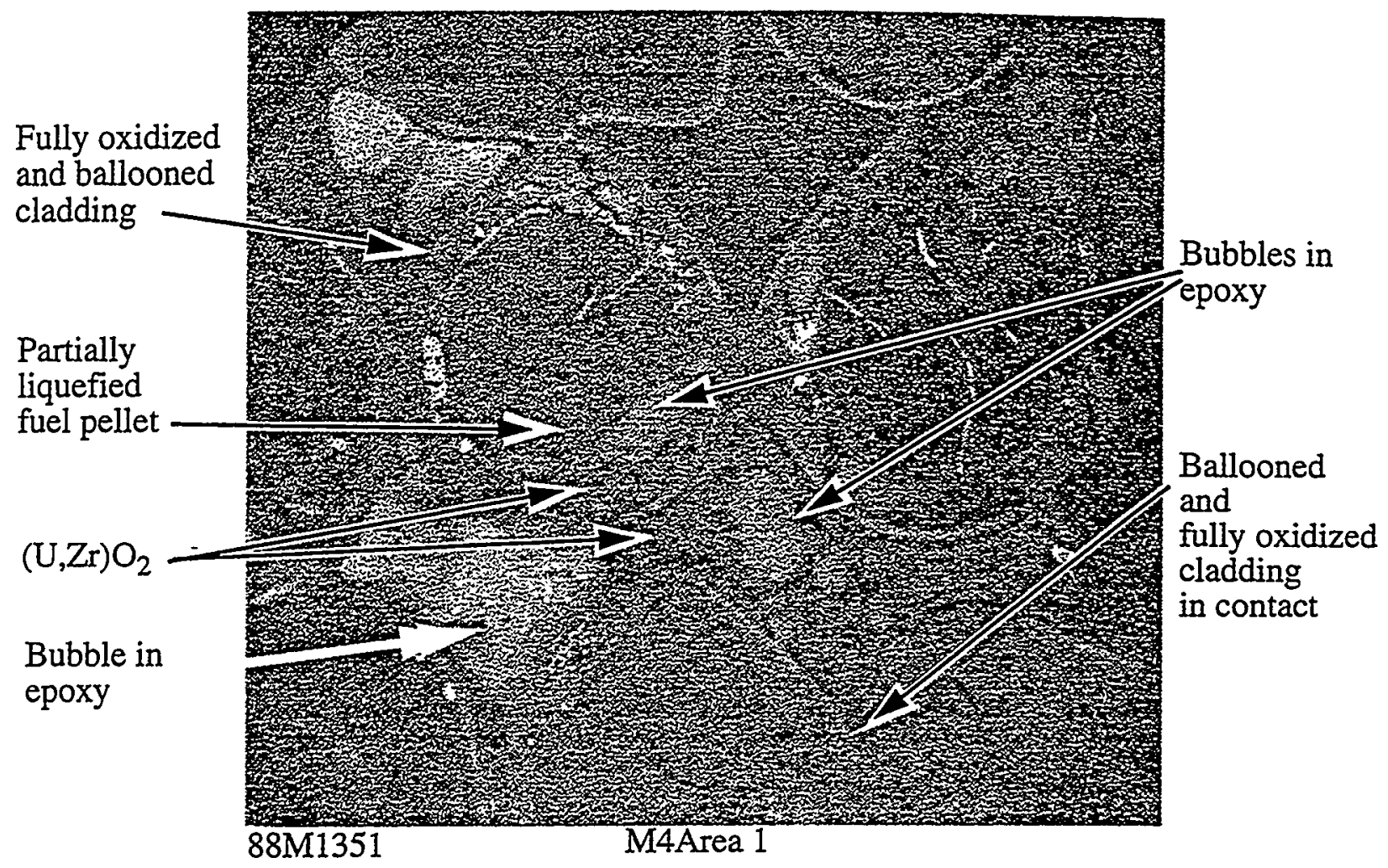

Figure 11. Example of asymmetric configuration of liquefied metallic layer from $1.04 \mathrm{~m}$ elevation of OECD LOFT FP-2 test bundle (M4Area1).

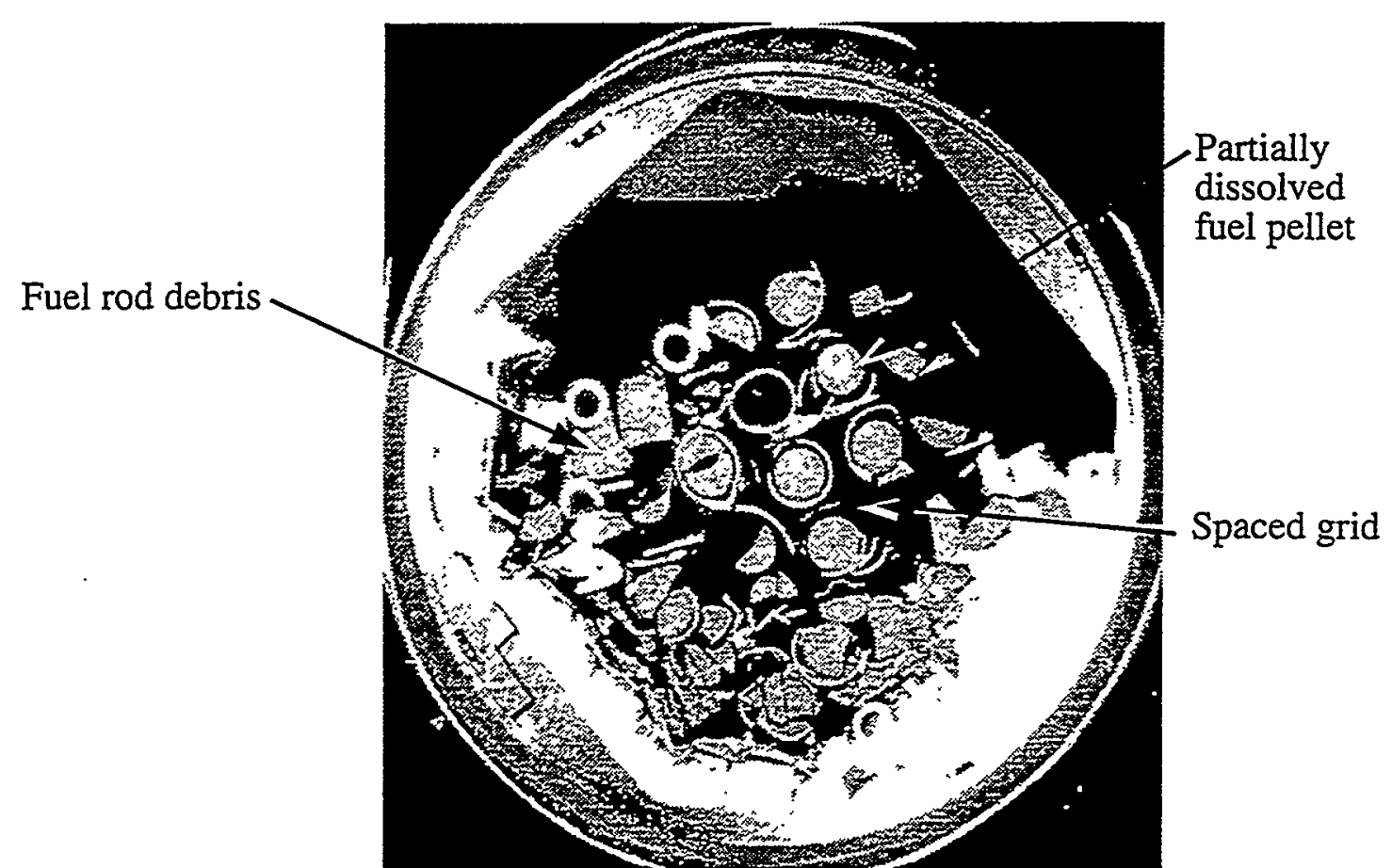

Figure 12. Example of asymmetric shape of liquefied cladding and dissolved fuel from PBF SFD 1-1 experiment. 
The presence of a large concentration of relocated material on the outer surface of an oxide layer may give it structural support and may effect the rate of oxidation of the underlying metallic layer. An example from the OECD LOFT FP-2 experiment ${ }^{5}$ of fully oxidized cladding surrounded by relocated material is shown in Figure 13. This figure indicates that partially oxidized cladding continued to oxidize after being

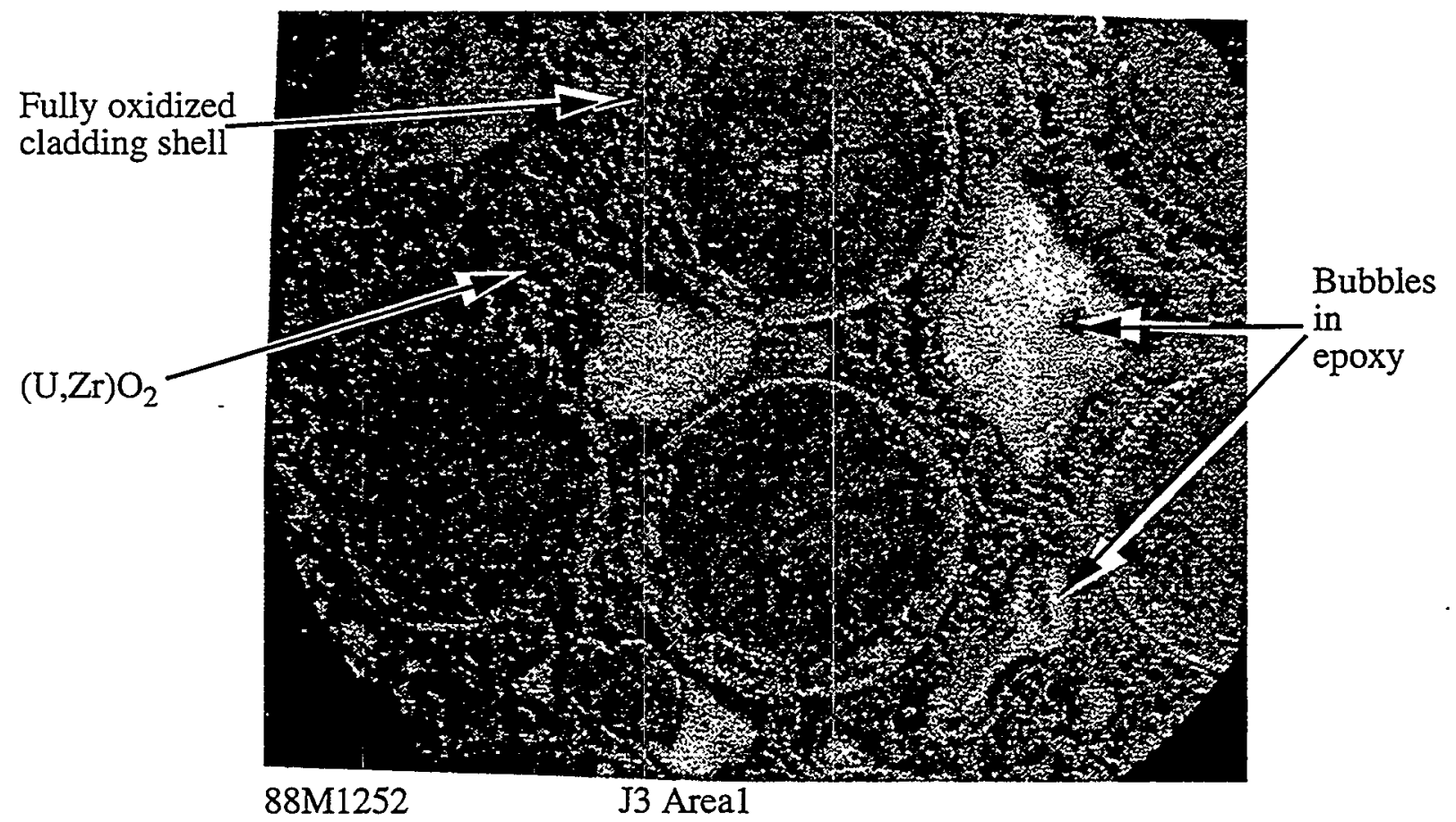

Figure 13. Example from OECD LOFT FP-2 experiment of fully oxidized cladding surrounded by relocated material.

surrounded by a large concentration of relocated material and that the relocated material reduced the stresses in the oxide layer or provided structural strength to the oxide layer. The figure and the calculation results thus indicate that in a region with a large amount of relocated material the code is underpredicting the capability of the oxide layer to retain the liquefied metallic cladding and/or overpredicting the blockage to oxidation of the intact cladding caused by the presence of relocated material.

\section{Rules for Calculating Configuration and Integrity of Cladding}

The PIE results indicate that the configuration and integrity of the cladding should be prescribed as a function of a damage index as defined in Table 1 of Section 1 and as shown in Figure 3 though Figure 6 of Section 1. According to the rules defined in Table 1 and Figure 3 through Figure 6, the metallic part of the cladding maintains an annular configuration when the damage progression is limited to no ballooning of the cladding or the metallic part of the cladding has not melted. Otherwise, the metallic part of the cladding is in the shape of a segment of an annulus, or, in other words, in the shape of a quarter moon. The melted metallic part of the cladding reacts with the oxidic part of the cladding and dissolves it in an asymmetrical manner. If high cladding temperatures are maintained and a significant temperature gradient exists across the oxidic part, then the oxidic part may fail due to excessive stress or dissolve to the vanishing point at some point on its circumference for the case of steam-starved conditions. Then, the melted part of the cladding slumps in the configuration of drops. 
This section defines the numerical values needed in the rules for cladding configuration and defines the equation for evaluating the integrity of the cladding. Section 4.1 defines the rules for mapping the configuration of the cladding after the metallic part has melted. Section 4.2 describes rules for defining the behavior of partially oxidized cladding after relocated material from above has frozen onto the external surface of the cladding. Section 4.3 presents the equations for calculating the thickness of the oxide layer accounting for its dissolution and thinning due to chemical reaction with the adjacent metallic layer and thickening due to oxidation. Section 4.4 presents the empirical equation used to calculate the failure of the oxide layer and the corresponding release of melted cladding that had been retained by the oxide layer. Section 4.5 revises the criterion for double-sided oxidation so it conforms better with theoretical and experimental results.

\subsection{Circumferential Relocation of Melted Cladding}

The PIE results presented in Section 3 indicate that melted metallic cladding relocates in the circumferential direction and changes in configuration from an annulus to a shape approximating that of a quarter moon. If cladding ballooning has not occurred, this change in configuration does not occur. The $\mathrm{PIE}$ results indicate that rules can be established for mapping the change in configuration of the cladding after the metallic part has melted. These rules are described as follows. On average, a liquefied metallic layer relocates in the circumferential direction so that it is concentrated in a $225^{\circ}$ segment of the circumference. After circumferential relocation of the cladding has occurred, oxidation occurs on only the outer surface of the relocated metallic layer. Circumferential relocation causes the inner surface of the metallic layer to be in contact with fuel and thus blocked from contact with steam. Figure 14 describes the model for circumferential relocation. After melting of the metallic layer, oxidation occurs only over a $225^{\circ}$ circumferential segment of the outer surface of the metallic layer. Also, a chemical reaction occurs between the metallic and oxidic parts of the cladding at their interface. This chemical reaction results in an asymmetrical dissolution of the oxidic part of the cladding. For cladding that has not ballooned or for metallic cladding with a temperature less than its liquefaction temperature, the metallic layer remains annular in configuration.

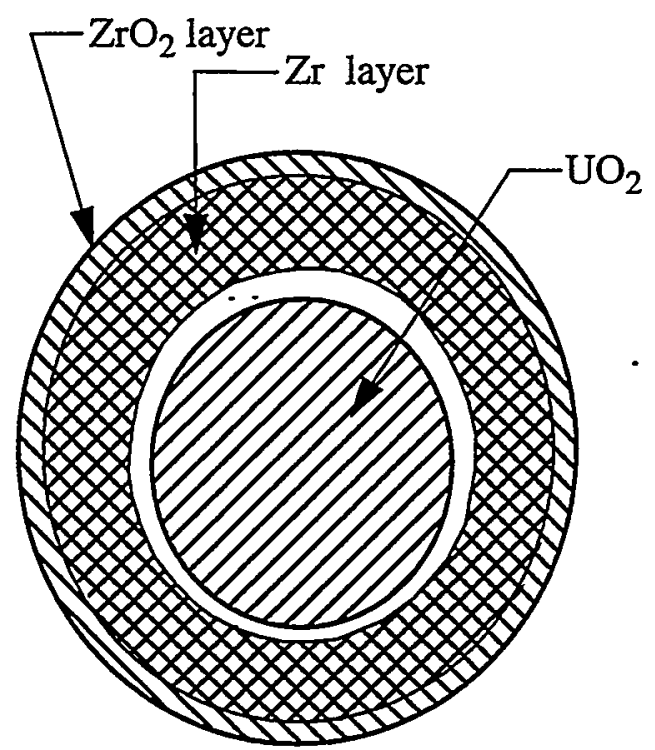

Configuration of $\mathrm{Zr}$ layer before melting

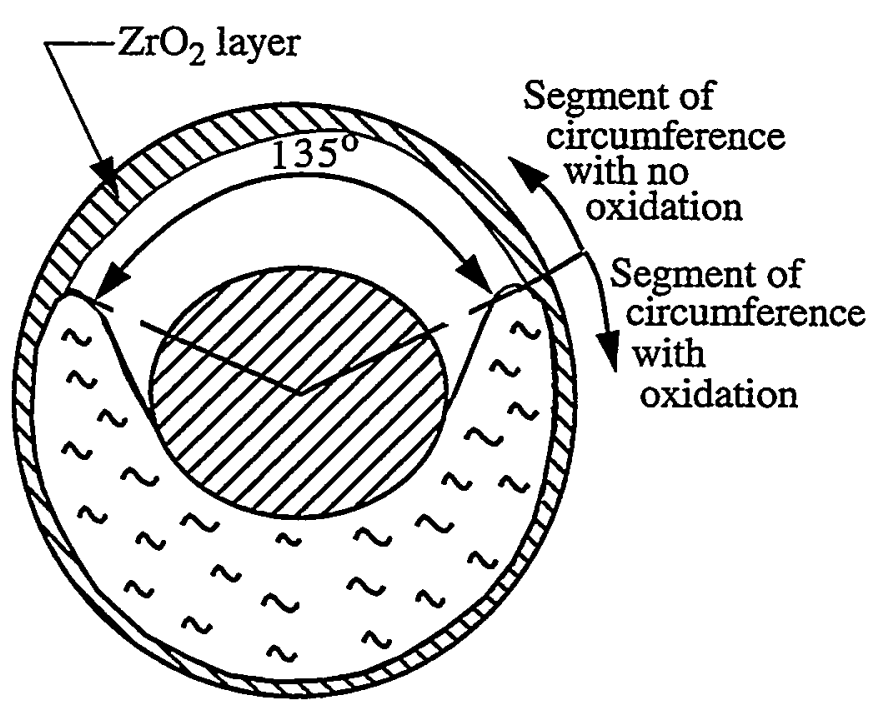

Configuration of $\mathrm{Zr}$ layer after melting

Figure 14. Configuration change resulting from melting of metallic part of cladding. 


\subsection{Behavior of Cladding at Locations with Relocated Material}

The presence of a large concentration of relocated material on the outer surface of the cladding changes the thermal, oxidation and structural behavior of the cladding. This section defines these changes in behavior based upon the PIE results presented in Section 3.

The PIE results indicate that the presence of relocated material leads to an increase in the integrity of the oxidic part of the cladding. Also, the presence of some material does not cause a total blockage of steam for the oxidation of the metallic part of the cladding. The increase in integrity may be due to several factors. First, there is a decrease in the temperature gradient across the cladding that is caused by the relocated material as it cuts off the cladding surface from convective heat transfer. As shown in Section 4.4, this reduction in temperature gradient decreases the stresses on the cladding oxide layer. Second, the relocated material may be oxidizing and generating heat with the result that the temperature gradient across the oxide layer is further reduced. Third, the integrity may be enhanced due to relocated material bridging fuel rods and thus providing radial support to the oxide layer. The continued ingress of steam for oxidation after the presence of some relocated material may be due to diffusion through the relocated material or by turbulent mass transport. A mechanistic model to calculate how the cladding integrity and oxidation are effected by relocated material is not an appropriate option; a three-dimensional calculation (axial, radial, and circumferential) of heat transfer, oxygen diffusion, and deformation would be required. So a set of rules for defining the behavior of the cladding will be established that result in the behavior of the cladding conforming to PIE results. The set of rules will establish the behavior of the cladding as a function of the concentration of relocated material at the external surface of the cladding. The concentration of relocated material will be measured by the fraction of local as-fabricated flow area (area external to fuel rods) that is filled with relocated material.

The first rule in the set of rules for defining cladding behavior imposes a boundary condition on the cladding that reduces its temperature gradient to the vanishing point and thus results in a vanishing of thermal stresses in the oxidic part of the cladding. This boundary condition is imposed when the concentration of relocated material exceeds a threshold value. The concentration of relocated material is represented by the symbol $f_{\text {fill }}$, where $f_{\text {fill }}$ is the fraction of flow area at the location of the cladding that is filled with relocated material. The threshold value is represented by the symbol $f_{b l k}$. If $f_{\text {fill }}>f_{b l k}$, then the temperature gradient across the cladding is assumed to be negligible. As will be shown in Section 4.4, this vanishing of temperature gradient results in zero stress in the oxidic part of the cladding, and thus this part of the cladding maintains its integrity and maintains its capability to retain the liquefied metallic part of the cladding. The value of $f_{b l k}$ can not be directly determined either by theoretical analysis or from experimental results. So it needs to be determined by a trial and error method. A value of $f_{b l k}$ will be guessed. The five experiments studied in Section 2 will be calculated and the axial distribution in cladding oxidation compared with the measured axial distribution in cladding oxidation. If a significant discrepancy exists, an adjustment will be made to the value of $f_{b l k}$. The value of $f_{b l k}$ is expected to be in the range of $0.5<\mathrm{f}_{\mathrm{blk}}<1.0$.

The second rule in the set of rules for defining behavior imposes structural boundary conditions for the cladding as a function of the concentration of relocated material. When the concentration of relocated material, represented by the symbol $f_{\text {fill }}$, exceeds the threshold value of $f_{\text {sup }}$, then a ceramic crust is defined to form at the bottom of the relocated material that supports the possibly liquefied relocated material above it. This rule is supported by the PIE results of PBF SFD 1-4 test, ${ }^{3}$ which indicated that a large 
concentration of material at a temperature of $2800 \mathrm{~K}$ slumped into the elevation interval of $0.10 \mathrm{~m}$ to 0.30 $\mathrm{m}$. This rule is also supported by the investigation of the behavior of relocated melted cladding performed by Veshchunov and Palagin. ${ }^{14}$ The ceramic crust will be defined to support metallic melted material until the ceramic crust attains its melting temperature. A crust with this characteristic can form by oxidation of the bottom surface of the relocated material. A schematic of the cladding configuration imposed by this rule is shown in Figure 15. The relocated material is defined to not relocate further until the temperature at

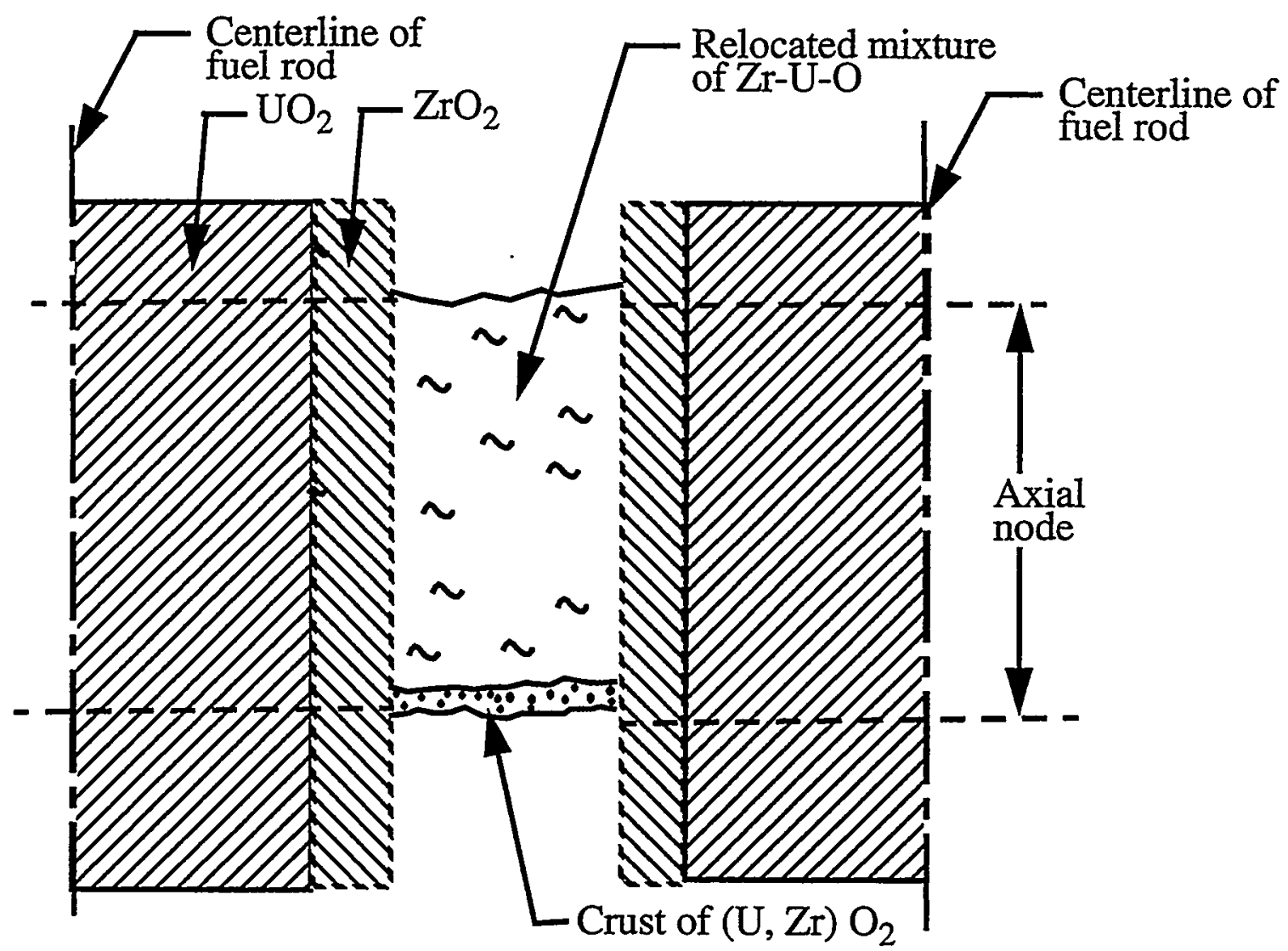

Figure 15. Crust formation at bottom surface of large concentration of relocated material.

that location exceeds the solidification temperature of the ceramic crust.

The third rule in the set of rules for cladding behavior imposes a boundary condition on the oxidation of the cladding as a function of the concentration of relocated material at a location. The oxidation of intact cladding is assumed to proceed unabated by the presence of relocated material until the fraction of asfabricated flow area filled with relocated material is greater than a threshold value. This threshold value is represented by the symbol $f_{a c}$. Thus, for $f_{\text {fill }}>f_{a c}$, the blockage is assumed to prevent oxidation from occurring. For $\mathrm{f}_{\mathrm{fill}}<\mathrm{f}_{\mathrm{ac}}$, the amount of steam available for oxidation is assumed to be the same as would be available in the absence of a blockage. This rule is based upon PIE results presented in Section 3 that show complete oxidation of cladding at locations with a large concentration of relocated material and where these locations are positioned such that complete oxidation could not have occurred before relocated material was present. The value of $f_{a c}$ can not be directly determined either by theoretical analysis or from experimental results. So it needs to be determined by a trial and error method. A values of $f_{a c}$ will be guessed. The five experiments studied in Section 2 will be analyzed and the calculated axial distribution in 
fraction of cladding oxidation compared with the measured axial distribution in fraction of cladding oxidation. If a significant discrepancy exists, an adjustment will be made to the value of $f_{a c}$. The value of $\mathrm{f}_{\mathrm{ac}}$ is expected to be in the range of $0.5<\mathrm{f}_{\mathrm{ac}}<1.0$.

\subsection{Dissolution and Thinning of Oxide Layer}

This section describes models for calculating the thinning of the oxide layer on fuel rod cladding by dissolution into the metallic part of the cladding that is in contact with the oxide layer. The models are to some extent based on the model for $\mathrm{ZrO}_{2}$ dissolution developed by Wilhelm and Garcia ${ }^{15}$, which calculates the dissolution of the oxide layer in a continuum composed only of $\mathrm{Zr}$ and $\mathrm{ZrO}_{2}$. Another model for $\mathrm{ZrO}_{2}$ dissolution developed by Veshchunov et. al. ${ }^{16}$ has also been developed. This model calculates the dissolution of $\mathrm{ZrO}_{2}$ in a continuum composed of $\mathrm{UO}_{2}, \mathrm{Zr}$, and $\mathrm{ZrO}_{2}$. This model has the benefit of accounting for the affect of $\mathrm{UO}_{2}$ dissolution on $\mathrm{ZrO}_{2}$ dissolution, but it also has the disadvantage of requiring a complex numerical solution using diffusion coefficients that may not be accurately known for a broad range of conditions. The Wilhelm and Garcia model, but not the Veshchunov model, can be implemented within the defined scope of effort.

The Wilhelm and Garcia model is based on experimental data that showed the dissolution followed an approximately parabolic kinetics relation after a short incubation period. ${ }^{17}$ During the incubation period, the experimental results showed that there was a rapid dissolution of $\mathrm{ZrO}_{2}$ by the $\mathrm{Zr}$. At the end of the incubation period, the mass fraction of $\mathrm{ZrO}_{2}$ in the $\mathrm{Zr}-\mathrm{O}$ mixture was 0.233 . The incubation occurred over a $14 \mathrm{~s}$ interval of time.

After the incubation period, the dissolution and thinning of the oxide layer followed in an approximate manner the parabolic kinetics law. According to this law, the rate of thinning of the oxide layer is given by the equation

$$
\frac{\mathrm{d} \delta}{\mathrm{dT}}=\frac{\mathrm{A}}{\delta} \mathrm{e}^{\left(\frac{-\mathrm{B}}{\mathrm{RT}}\right)}
$$

where

$$
\begin{array}{lll}
\delta & = & \text { depth of dissolution of } \mathrm{ZrO}_{2} \text { layer }(\mathrm{m}), \\
\mathrm{t} & = & \text { time }(\mathrm{s}) \\
\mathrm{T} & = & \text { temperature }(\mathrm{K}), \\
\mathrm{A}, \mathrm{B} & = & \text { parabolic rate constants determined from experimental data, } \\
\mathrm{R} & = & \text { gas constant }\left(8.314 \frac{\mathrm{J}}{\mathrm{mol}} \cdot \mathrm{K}\right) .
\end{array}
$$

For a constant temperature, this equation can be integrated over a time interval to give

$$
\delta_{2}^{2}-\delta_{1}^{2}=2 A e^{\left(\frac{-B}{R T}\right)} \Delta t
$$


where

$$
\begin{array}{lll}
\delta_{2} & = & \text { depth of dissolution at end of time interval (m), } \\
\delta_{1} & = & \text { depth of dissolution at beginning of time interval (m), } \\
\Delta \mathrm{t} & = & \text { time interval (s). }
\end{array}
$$

The value of $\delta$ is equal to zero at the instant the incubation period is complete.

According to Wilhem and Garcia, the constants $\mathrm{A}$ and $\mathrm{B}$ in the above equation have the following values,
$\mathrm{A} \quad=\quad 3.35 \times 10^{12}$
$\mathrm{B} \quad=\quad 8.974 \times 10^{5}$

In the first approach to modeling the dissolution of the $\mathrm{ZrO}_{2}$ layer, the Wilhelm and Garcia dissolution model will be implemented as follows. First, the time at which the metallic part of the cladding has completely melted will be stored and the time at the end of the incubation period for dissolution will be identified.

Thus,

$t_{c}=t_{m}+t_{c u b}$

where

$$
\begin{array}{lll}
t_{c} & = & \text { time at which the incubation period for dissolution is complete at a location (s), } \\
t_{m} & = & \text { time at which the metallic part of the cladding was completely melted }(s), \\
t_{c u b} & = & \text { period of time for incubation dissolution }(s) .
\end{array}
$$

As indicated from experimental results, the value of $\mathrm{t}_{\mathrm{cub}}$ is $14 \mathrm{~s}$.

Next, beginning at the time of $t_{c}$ at the location where incubation dissolution occurred, the reduction in thickness due to dissolution will be calculated.

According to the experimental results examined by Wilhelm and Garcia, the mass fraction of $\mathrm{ZrO}_{2}$ in the liquefied $\mathrm{Zr}-\mathrm{O}$ mixture is equal to 0.233 . Thus,

$0.233=\frac{\pi\left(\rho_{o x} r_{c} h_{o x}\right)}{\pi\left(\rho_{o x} r_{c} h_{o x}\right)+\pi \rho_{z r} r_{c}\left(h_{o}-G h_{o x}\right)}$

where

$$
\begin{array}{lll}
\rho_{\mathrm{ox}} & = & \text { density of } \mathrm{ZrO}_{2}\left(\mathrm{~kg} / \mathrm{m}^{3}\right), \\
\mathrm{r}_{\mathrm{c}} & = & \text { radius to midplane of cladding }(\mathrm{m}), \\
\mathrm{h}_{\mathrm{ox}} & = & \text { thickness of oxide layer dissolved during incubation period }(\mathrm{m}),
\end{array}
$$




$$
\begin{array}{lll}
\rho_{z \mathrm{r}} & = & \text { density of } \mathrm{Zr}\left(\mathrm{kg} / \mathrm{m}^{3}\right), \\
\mathrm{G}^{-1} & = & \text { Pilling-Bedworth ratio of } \mathrm{ZrO}_{2}(1.5)^{18}, \\
\mathrm{~h}_{\mathrm{o}} & = & \text { as-fabricated thickness of cladding (m). }
\end{array}
$$

Solving the above equation for $h_{\mathrm{ox}}$, the result is

$$
h_{o x}=\frac{0.233 \rho_{z:} h_{o}}{0.767 \rho_{o x}+0.233 G \rho_{z r}}
$$

The thickness of the oxide layer at the end of the incubation dissolution period is then calculated by the equation

$$
\mathrm{d}_{\mathrm{ox}}=\mathrm{d}_{\mathrm{oxi}}-\mathrm{h}_{\mathrm{ox}}^{-}
$$

where

$$
\begin{aligned}
& \mathrm{d}_{\mathrm{ox}}=\quad \text { thickness of oxide layer at a location at time of } \mathrm{t}_{\mathrm{c}}(\mathrm{m}) \text {, } \\
& \mathrm{d}_{\mathrm{oxi}}=\quad \text { thickness of oxide layer at a location at time of } \mathrm{t}_{\mathrm{c}} \text { not accounting for dissolution } \\
& \text { during incubation period }(\mathrm{m}) \text {. }
\end{aligned}
$$

For typical values of $r_{c}$ and $h_{0}$, the above equations calculate that the oxide layer is completely dissolved at any location at which the cladding is less than $19 \%$ oxidized at the time that the metallic part of the cladding at that location is completely liquefied.

For a time greater than $t_{c}$, the cumulative of dissolution is calculated by the integration of the parabolic kinetics equation over a time step. The change in temperature during a time step is assumed to be negligible. Applying the results of integration shown in Equation (2).

$\delta_{2}=\left[\delta_{1}^{2}+2 \mathrm{Ae}^{\left(\frac{-\mathrm{B}}{\mathrm{RT}}\right)} \Delta \mathrm{t}\right]^{0.5}$

where

$\delta_{2}^{2}=\quad$ cumulative depth of dissolution at a location at end of time step (m),

$\delta_{1}^{2}=\quad$ cumulative depth of dissolution at a location at start of time step $(\mathrm{m})$,

$\Delta t \quad=\quad$ time step $(\mathrm{s})$,

$\mathrm{A}, \mathrm{B}=\quad$ constants defined in Equation (2).

For a time greater than $t_{c}$, the cumulative increase in oxidation is calculated by the equation

$\mathrm{d}_{\text {oxc2 }}=\left[\mathrm{d}_{\mathrm{oxci}}^{2}+2 \mathrm{Ce}{ }^{\left(\frac{-D}{\mathrm{~T}}\right)} \Delta \mathrm{t}\right]^{0.5}$ 
where

$$
\begin{array}{lll}
d_{\text {oxc2 }}= & \text { cumulative increase in oxide thickness at end of time step }(m), \\
d_{\text {oxcl }}= & \text { cumulative increase in oxide thickness at start of time step }(m), \\
C, D= & \text { coefficients in parabolic kinetics equation for oxidation }{ }^{1}, C=1.035 \times 10^{-6}, \\
& D=1.6014 \times 10^{4} .
\end{array}
$$

In the above equation, $d_{o x 1}$ is not reduced to account for the dissolution of the oxide layer. The parabolics kinetics equation for oxidation is based upon cumulative weight gain of oxygen in the cladding. The cumulative weight gain is not effected by dissolution.

The oxide thickness accounting for dissolution and oxidation is calculated by the equation

$$
\mathrm{d}_{\mathrm{ox2}}=\mathrm{d}_{\mathrm{ox1}}-\delta_{2}+\delta_{1}+\mathrm{d}_{\mathrm{oxc2} 2}-\mathrm{d}_{\mathrm{oxc1}}
$$

where

$$
\begin{array}{lll}
\mathrm{d}_{\mathrm{ox} 2}= & \begin{array}{l}
\text { oxide thickness at end of time step at location of cladding with adjacent metallic } \\
\text { and oxidic layers }(\mathrm{m}),
\end{array} \\
\mathrm{d}_{\mathrm{ox} 1}= & \text { same as } \mathrm{d}_{\mathrm{ox} 2} \text { but for start of time step }(\mathrm{m}) .
\end{array}
$$

The dissolution of the oxide layer will be assumed to not occur when the amount of oxygen in the melted part of the cladding is equal to or greater than the amount defined by the liquidus line of the $\mathrm{Zr}-\mathrm{U}$ $O$ phase diagram.

The cladding configuration calculated by the models for dissolution and oxidation is shown in Figure 16. A schematic of the change in oxide thickness during a time step is shown in Figure 17.

An alternative approach to calculating the depth of dissolution of the oxide layer will be investigated. This approach is based on the concept that the dissolution of the oxide layer proceeds at a rate that is some fraction of the dissolution of the fuel on the other side of the metallic layer. This approach has the virtue of more directly accounting for the effect of fuel dissolution on cladding oxide dissolution. The kinetics equations ${ }^{19}$ for the depth of dissolution of $\mathrm{UO}_{2}$ and $\mathrm{ZrO}_{2}$ immediately after the end of the incubation period are;

$$
\begin{aligned}
& \Delta \mathrm{d}_{\mathrm{u}}=\left[3.85 \times 10^{17} \mathrm{e}^{\frac{-1.067 \times 10^{6}}{\mathrm{RT}}} \Delta \mathrm{t}\right]^{0.5} \\
& \Delta \mathrm{d}_{\mathrm{z}}=\left[3.35 \times 10^{12} \mathrm{e}^{\frac{-8.97 \times 10^{5}}{\mathrm{RT}}} \Delta \mathrm{t}\right]^{0.5}
\end{aligned}
$$

where 


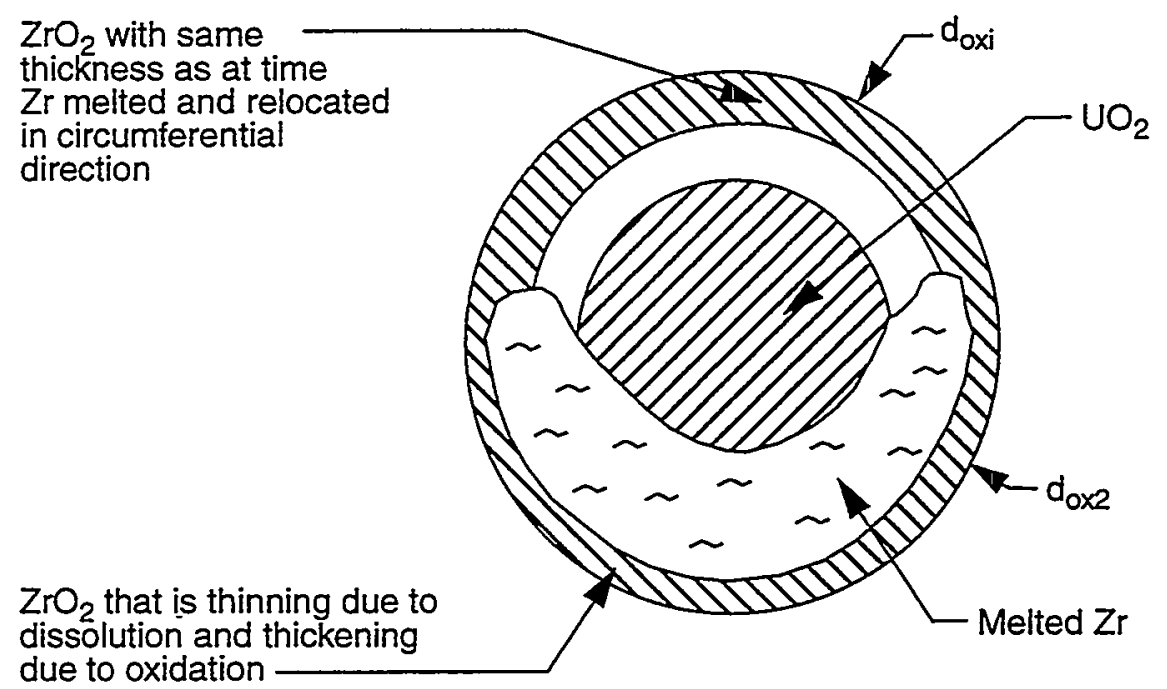

Figure 16. Cladding configuration calculated by models for dissolution and oxidation.

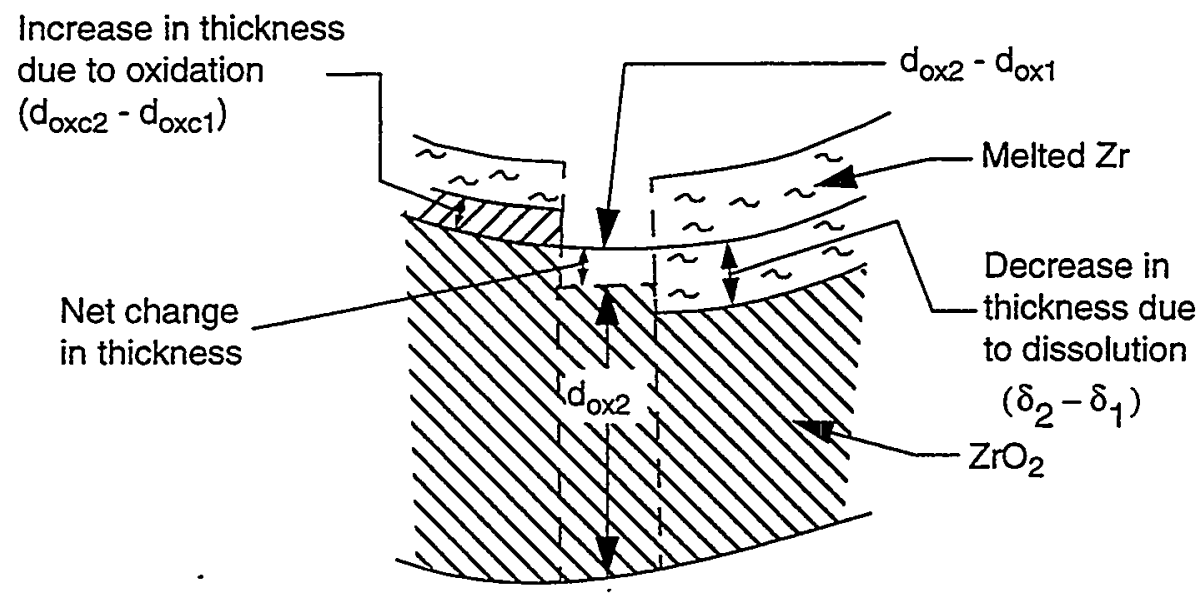

Figure 17. Schematic of change in thickness of cladding oxide layer during time step.

\begin{tabular}{|c|c|c|}
\hline$\Delta \mathrm{d}_{\mathrm{u}}$ & $=$ & $\begin{array}{l}\text { depth of dissolution of } \mathrm{UO}_{2} \text { during time interval of } \Delta \mathrm{t} \text { at beginning of period } \\
\text { for which the parabolic kinectics equation is applicable }(\mathrm{m}) \text {, }\end{array}$ \\
\hline$\Delta d_{z}$ & $=$ & $\begin{array}{l}\text { depth of dissolution of } \mathrm{ZrO}_{2} \text { during time interval of } \Delta \mathrm{t} \text { at beginning of period } \\
\text { for which the parabolic kinetics equation is applicable }(\mathrm{m}) \text {, }\end{array}$ \\
\hline$\Delta \mathrm{t}$ & $=$ & time step (s), \\
\hline$T$ & $=$ & temperature $(\mathrm{K})$ \\
\hline $\mathrm{R}$ & $=$ & gas constant $(8.314 \mathrm{~J} / \mathrm{mol} \cdot \mathrm{K})$. \\
\hline
\end{tabular}


According to the theoretical results of Veshchunov ${ }^{16}$, the above equation for calculating the depth of dissolution of the $\mathrm{UO}_{2}$ may overpredict the depth of dissolution by a factor of two or more. This discrepancy is due to the rate of dissolution equation for $\mathrm{UO}_{2}$ being based on experimental results that did not account for simultaneous dissolution of $\mathrm{ZrO}_{2}$ layer. So an adjustment may be required to the equation for calculating the depth of dissolution of the $\mathrm{UO}_{2}$, but no adjustment will be made unless identified as being required by model testing and assessment.

After the incubation period, the rate of dissolution of $\mathrm{UO}_{2}$ by $\mathrm{Zr}$ is faster than the rate of dissolution $\mathrm{ZrO}_{2}$ by $\mathrm{Zr}$. The depths of dissolution for $\mathrm{UO}_{2}$ and $\mathrm{ZrO}_{2}$ for a range of temperatures and a time interval of $0.1 \mathrm{~s}$ are shown in Table 7. The depths of dissolution were calculated assuming no prior dissolution beyond the incubation period and assuming no interaction between the dissolution of $\mathrm{UO}_{2}$ and $\mathrm{ZrO}_{2}$. Also, the dissolution was not limited at the liquidus or solidus boundaries of the phase diagram for a mixture of U$\mathrm{Zr}-\mathrm{O}$. The rate of dissolution of the $\mathrm{UO}_{2}$ is a factor of four or greater than the rate of dissolution of the $\mathrm{ZrO}_{2}$. As shown in Table 3, the rate of dissolution of $\mathrm{UO}_{2}$ relative to that of $\mathrm{ZrO}_{2}$ increases with temperature.

Table 7. Comparison of depths of dissolution of $\mathrm{UO}_{2}$ and $\mathrm{ZrO}_{2}$ for case of no prior dissolution and time interval of $0.1 \mathrm{~s}$.

\begin{tabular}{|c|c|c|}
\hline Temperature & $\begin{array}{c}\mathrm{UO}_{2} \text { Depth of } \\
\text { dissolution }\end{array}$ & $\begin{array}{c}\mathrm{ZrO}_{2} \text { depth of } \\
\text { dissolution }\end{array}$ \\
\hline$(\mathrm{K})$ & $(\mathrm{mm})$ & $(\mathrm{mm})$ \\
\hline \hline 2200 & 0.04 & 0.01 \\
\hline 2300 & 0.18 & 0.04 \\
\hline 2400 & 0.48 & 0.10 \\
\hline 2500 & 1.40 & 0.25 \\
\hline 2600 & 3.75 & 0.56 \\
\hline
\end{tabular}

In this alternative approach, the depth of dissolution of the $\mathrm{ZrO}_{2}$ layer is calculated by the following four equations. First,

$\mathrm{f}_{\mathrm{zu}}=\Delta \mathrm{d}_{\mathrm{z}} / \Delta \mathrm{d}_{\mathrm{u}}$

where

$\mathrm{f}_{\mathrm{zu}}=\quad$ ratio of rate of dissolution of $\mathrm{ZrO}_{2}$ to $\mathrm{UO}_{2}$ at current temperature.

The depth of dissolution of the $\mathrm{UO}_{2}$ during the time step is then calculated by the equations

$$
\begin{aligned}
\mathrm{d}_{\mathrm{u} 2} & =\left[\mathrm{d}_{\mathrm{U} 1}^{2}+7.70 \times 10^{17} \mathrm{e}^{\left(-1.067 \times 10^{6} / \mathrm{RT}\right)} \Delta \mathrm{t}\right]^{0.5} \\
\Delta \mathrm{d}_{\mathrm{u}} & =\mathrm{d}_{\mathrm{u} 2}-\mathrm{d}_{\mathrm{u} 1}
\end{aligned}
$$


where

$$
\begin{array}{lll}
\mathrm{du}_{2} & = & \text { cumulative depth of dissolution at end of time step (m), } \\
\Delta \mathrm{d}_{\mathrm{u}} & = & \text { increment of dissolution during time step (m). }
\end{array}
$$

If the state of the $\mathrm{Zr}-\mathrm{U}-\mathrm{O}$ mixture as indicated by its phase diagram is outside the liquid regime, the increment of dissolution of $\mathrm{UO}_{2}$ during the time step is equal to zero.

The increment of dissolution of $\mathrm{ZrO}_{2}$ during the time step is calculated by the equation

$\delta_{2}-\delta_{1}=f_{z u} \Delta d_{u}$

A more mechanistic calculation of the thickness of the oxide layer may be possible with a model proposed for implementation in the SCDAP/RELAP5 code in the near future. This proposed model is designed to replace the existing parabolic kinetics equations for dissolution and oxidation with a simultaneous calculation of dissolution and oxidation that is based on the integral diffusion method. ${ }^{18,20,21}$ When this method is implemented and extended for application to melted metallic cladding interfacing with $\mathrm{UO}_{2}$ at its inner surface, the transient oxide thickness, represented by the variable $\mathrm{d}_{\mathrm{ox} 2}$, would be directly calculated, and the equations shown above to calculate $\delta_{2}$ and $\mathrm{d}_{\mathrm{oxc} 2}$ would not be needed.

\subsection{Failure of Oxide Layer}

The integrity of the oxide layer is determined by comparing the maximum stress in the oxide layer with the failure stress of the oxide layer. Stresses are generated in the oxide layer by a temperature gradient across the oxide layer. If the thickness of the oxide layer is not uniform in the circumferential direction, then a concentration of stress occurs at the point on the circumference with the minimum thickness. This concentration occurs because the force induced in the hoop direction by thermal stress from a temperature gradient must balance at all points on the circumference. When the stress exceeds the failure stress, the oxide layer at that point is regarded as no longer capable of retaining the melted metallic part of the cladding at that point. A breach is assumed to occur in the oxide layer and the melted cladding slumps through the breach. The current model for cladding failure uses two empirically determined constants and calculates failure based on the cladding temperature and extent of oxidation. The proposed model for cladding failure applies one empirically determined constant to a mechanistic calculation of the stresses in the oxide layer.

The current model in SCDAP/RELAP5/MOD3.2 for calculating the structural strength of the oxide layer is a simple model with two empirical constants. ${ }^{10}$ The model is similar to that used by other severe accident analysis computer codes such as the ICARE2 code ${ }^{11,12}$ developed in France and the SEFDAN code ${ }^{13}$ developed in Japan. The model is described by the equation

$f_{s}=1 ; T_{\text {clad }}<T_{\text {fal }}$ or $\left(f_{\text {ox }}>f_{\text {thr }}\right.$ and $\left.T_{\text {clad }}<T_{\text {mox }}\right)$

$f_{s}=0$; conditions other than those for $f_{s}=1$ 
where

$\mathrm{f}_{\mathrm{s}}=\quad$ indicator of structural strength of the oxide layer; $1=$ structural strength is sufficient to retain liquefied material, $0=$ structural strength not sufficient to retain liquefied material,

$\mathrm{T}_{\text {clad }}=$ temperature of oxide layer $(\mathrm{K})$

$\mathrm{f}_{\mathrm{ox}}=$ fraction of oxidation of cladding,

$\mathrm{T}_{\text {fal }} \quad=\quad$ user-defined temperature $(\mathrm{K})$ at which the oxide layer fails when this temperature is exceeded and the fraction of oxidation is less than the user-defined threshold value of $f_{t h r}$ The default values for $T_{\text {fal }}$ and $f_{\text {thr }}$ are $2500 \mathrm{~K}$ and 0.6 , respectively,

$\mathrm{T}_{\operatorname{mox}}=\quad$ melting temperature of oxide layer in contact with $\operatorname{Zr}(2830 \mathrm{~K})$.

If $\mathrm{T}_{\text {clad }}>\mathrm{T}_{\mathrm{mox}}$, then that location of the fuel rod begins the transition in configuration from that of an intact rod to that of a molten pool. In this case, liquefied material is assumed to not slump down the surface of fuel rods.

The proposed model for cladding failure compares the stresses in the oxide layer with the failure stress of the oxide layer. Since there is an order of magnitude uncertainty in the failure stress of the oxide layer and also large uncertainties in other structural properties, an empirically determined constant is also needed with this model.

The model developed by Boldyrev ${ }^{22}$ et al will be used to calculate the stresses in the oxide layer. According to this model, the maximum circumferential stress in an oxide layer is calculated by the equation

$$
\sigma=c \alpha \Delta T E \frac{d_{0 \times 2}^{2}}{\left(d_{0 \times 2}-\delta_{2}\right)^{2^{2}}}
$$

where

\begin{tabular}{|c|c|c|}
\hline$\sigma$ & $=$ & maximum circumferential stress in oxide layer $(\mathrm{Pa})$, \\
\hline$\alpha$ & $=$ & coefficient of thermal expansion of oxide layer $(1 / \mathrm{K})$, \\
\hline$\Delta \mathrm{T}$ & $=$ & $\begin{array}{l}\text { difference in temperature between inside and outside surfaces } \\
\text { of oxide layer }(\mathrm{K}) \text {, }\end{array}$ \\
\hline$E$ & $=$ & modulus of elasticity of oxide layer $(\mathrm{Pa})$ \\
\hline$d_{o x 2}$ & $=$ & thickness of oxide layer not accounting for fuel dissolution (m), \\
\hline$\delta_{2}$ & $=$ & depth of dissolution of oxide layer $(m)$ \\
\hline c & $=$ & empirically determined constant $(\sim 0.90)$. \\
\hline
\end{tabular}

The value of $\Delta T$ in the above equation will be large at a location where quench is occurring. As a result, failure of the oxide layer and the release of retained liquefied material is likely in the event of a 
quench of hot and partially oxidized cladding. Also, the above equation calculates the stresses to be very high at a location in which dissolution of the oxide layer reduces its thickness to the vanishing point.

The oxide layer will be considered to lose its structural integrity and release retained liquefied material when the following condition holds;

$\sigma>S_{u}$

where

$\mathrm{S}_{\mathrm{u}} \quad=\quad$ ultimate strength of oxide layer as determined by $\operatorname{MATPRO}^{19}(\mathrm{~Pa})$.

For an oxide temperature greater than $1869 \mathrm{~K}$, MATPRO assigns $\mathrm{S}_{\mathrm{u}}$ a constant value of $1 . \times 10^{6} \mathrm{~Pa}$, with an expected standard error of \pm 0.7 times the predicted value. Since a large amount of uncertainty exists in the value of $S_{u}$, the variable $c$ in the equation for stress will be set to a value that results in calculated timing for oxide failure being in agreement with the measured results. The preliminary value of $\mathrm{c}$ is 0.90 .

For a typical severe accident scenario at a location that is not steam-starved, the new model for calculating the integrity of the oxidic part of the cladding predicts that the oxide layer is vulnerable to failure in two ranges of temperature. The first range of temperature is the range of $2300 \mathrm{~K}$ to $2350 \mathrm{~K}$. The second range is the range from $2650 \mathrm{~K}$ to $2700 \mathrm{~K}$. These ranges of vulnerability are shown in Table 8 , where the ratio of the stress in the oxide layer to the ultimate stress (stress ratio) for severe accident conditions representative of the PBF SFD 1-1 experiment ${ }^{2}$ are shown. A stress ratio value greater than 1.0 is considered to result in a failure of the oxide layer. The symbols in the table correspond with the symbols in the equation for stress in the oxide layer. The values of the modulus of elasticity of the oxide layer were obtained from MATPRO. ${ }^{19}$ The MATPRO value of the coefficient of thermal expansion is a constant value of $1.30 \times 10^{-5}(1 / \mathrm{K})$ over the temperature range presented in Table 2 . Two ranges of vulnerability result because the dissolution of the $\mathrm{ZrO}_{2}$ layer temporarily stops due to saturation with oxygen of the melted layer and due to the stresses in the oxide layer being reduced because the modulus of elasticity of the oxide layer decreases with temperature. The dissolution attains a temporary maximum value in the temperature range of $2300 \mathrm{~K}$ to $2350 \mathrm{~K}$. Dissolution does not occur again until the cladding attains a temperature greater than the monotectic temperature of a $\mathrm{Zr}-\mathrm{U}-\mathrm{O}$ mixture $(-2700 \mathrm{~K})$, when the capability of the mixture to absorb oxygen increases significantly. As seen in Table 2, the oxide layer is calculated to not fail for cladding temperatures less than $2300 \mathrm{~K}$ and dissolution depths less than $25 \%$ of the maximum thickness of the oxide layer. For a temperature of $2700 \mathrm{~K}$ and a dissolution depth greater than $50 \%$ of the maximum thickness of the oxide layer, failure of the oxide layer is calculated to occur.

The scoping calculations presented in Table 8 indicate that the proposed model for evaluating cladding integrity may improve the calculated axial distribution in cladding oxidation. The calculated extent of cladding oxidation at the $0.35 \mathrm{~m}$ and $0.45 \mathrm{~m}$ elevations for the PBF SFD 1-1 test are shown in Table 9. The table also presents the measured extent of oxidation at these elevations. At the $0.45 \mathrm{~m}$ elevation, the calculated extent of oxidation with the proposed model is in significantly better agreement with the measured extent of oxidation than the current model. At the $0.35 \mathrm{~m}$ elevation, the extent of oxidation with the proposed model is somewhat overpredicted, while the current model significantly 
underpredicted the extent of oxidation. The implementation of other models proposed in this report is expected to further improve the calculation of the axial distribution in cladding oxidation.

At the $0.45 \mathrm{~m}$ elevation, the temperature at failure of the oxide layer was calculated to be greater than the maximum temperature at that location as determined from PIE results. This discrepancy may be due to the calculations not modeling the relocation of melted metallic cladding in the circumferential direction. This relocation reduces the surface area of oxidation and thus results in the rate of heatup being significantly reduced, which in turn may result in a greater extent of oxidation for a given maximum temperature.

Table 8. Stress ratio in cladding oxide layer for typical range of severe accident conditions.

\begin{tabular}{|c|c|c|c|c|c|c|c|c|c|}
\hline Time & Elev. & $\begin{array}{c}\text { Clad. } \\
\text { temp. }\end{array}$ & $\Delta \mathrm{T}$ & $\begin{array}{c}\mathrm{UO}_{2} \\
\text { depth } \\
\text { of } \\
\text { diss. }\end{array}$ & $\begin{array}{c}\mathrm{ZrO}_{2} \\
\text { thick. }\end{array}$ & $\begin{array}{c}\mathrm{ZrO}_{2} \\
\text { depth } \\
\text { of } \\
\text { diss. }\end{array}$ & $\mathrm{E}$ & $\begin{array}{c}\left(\mathrm{d}_{\mathrm{ox} 2}\right)^{2} / \delta^{2} \\
\left(\mathrm{~d}_{\left.\mathrm{ox} 2^{-} \delta_{2}\right)^{2}}\right.\end{array}$ & $\sigma / \mathrm{S}_{\mathrm{u}}$ \\
\hline$(\mathrm{s})$ & $(\mathrm{m})$ & $(\mathrm{K})$ & $(\mathrm{K})$ & $(\mathrm{mm})$ & $(\mathrm{mm})$ & $(\mathrm{mm})$ & $(\mathrm{pa})$ & - & \\
\hline \hline 2050 & 0.45 & 2228 & 0.86 & 0.049 & 0.176 & 0.011 & $0.467 \times 10^{11}$ & 1.15 & 0.55 \\
\hline 2070 & 0.45 & 2305 & 1.40 & 0.158 & 0.199 & 0.035 & $0.406 \times 10^{11}$ & 1.48 & 0.98 \\
\hline 2090 & 0.45 & 2366 & 1.62 & 0.177 & 0.217 & 0.037 & $0.356 \times 10^{11}$ & 1.45 & 0.98 \\
\hline 2110 & 0.45 & 2430 & 1.83 & 0.196 & 0.234 & 0.041 & $0.305 \times 10^{11}$ & 1.47 & 0.96 \\
\hline 2130 & 0.35 & 2230 & 1.01 & 0.045 & 0.188 & 0.011 & $0.466 \times 10^{11}$ & 1.13 & 0.62 \\
\hline 2130 & 0.45 & 2463 & 1.97 & 0.207 & 0.244 & 0.037 & $0.279 \times 10^{11}$ & 1.39 & 0.89 \\
\hline 2140 & 0.35 & 2332 & 1.59 & 0.168 & 0.220 & 0.037 & $0.384 \times 10^{11}$ & 1.45 & 1.03 \\
\hline 2140 & 0.45 & 2531 & 2.28 & 0.228 & 0.264 & 0.041 & $0.225 \times 10^{11}$ & 1.40 & 0.84 \\
\hline 2170 & 0.45 & 2632 & 2.88 & 0.260 & 0.294 & 0.039 & $0.143 \times 10^{11}$ & 1.33 & 0.64 \\
\hline 2190 & 0.45 & 2698 & 3.42 & 0.283 & 0.315 & 0.042 & $0.901 \times 10^{10}$ & 1.33 & 0.48 \\
\hline 2200 & 0.45 & 2730 & 3.73 & 1.55 & 0.326 & 0.230 & $0.645 \times 10^{10}$ & 11.7 & 3.28 \\
\hline
\end{tabular}

Table 9. Comparison of cladding oxidation for PBF SFD 1-1 test with current and proposed models for evaluating cladding integrity

\begin{tabular}{|c|c|c|c|}
\hline elevation & \multicolumn{3}{|c|}{ extent of cladding oxidation (\%) } \\
\hline$(\mathrm{m})$ & measured & current model & proposed model \\
\hline \hline 0.35 & 0.25 & 0.16 & 0.33 \\
\hline 0.45 & 0.65 & 0.36 & 0.56 \\
\hline
\end{tabular}


The variable $\Delta T$ in Equation 16 for $\sigma$ is not directly calculated by SCDAP; the code calculates the temperatures of the inside and outside surfaces of the cladding but not specifically the inside and outside temperatures of the oxide layer of the cladding. Nevertheless, the inside and outside temperatures of the oxide layer will be assumed to be same as the inside and outside temperatures of the cladding. Since the thermal conductivity of the $\mathrm{ZrO}_{2}$ part of the cladding is much less than that of the $\mathrm{Zr}$ part of the cladding, this assumption is appropriate for all but a $\mathrm{ZrO}_{2}$ layer that is thin relative to the $\mathrm{Zr}$ layer. In the case of a thin layer of $\mathrm{ZrO}_{2}$, it may be best to calculate the inside and outside temperatures of the oxide layer assuming steady state heat transfer and using the local nuclear heat and cladding oxidation heat generation. The equation used to solve for $\Delta \mathrm{T}$ will be

$$
q_{n}+q_{o x}=2 \pi r_{o x} k \frac{\Delta T}{d_{o x 2}}
$$

where

$\begin{array}{lll}\mathrm{q}_{\mathrm{n}} & = & \text { local nuclear heat generation rate }(\mathrm{W} / \mathrm{m}) . \\ \mathrm{q}_{\mathrm{ox}} & = & \text { local oxidation heat generation rate }(\mathrm{W} / \mathrm{m}), \\ \mathrm{r}_{\mathrm{ox}} & = & \text { radius of midplane of oxide layer }(\mathrm{m}), \\ \mathrm{k} & = & \text { thermal conductivity of oxide layer }(\mathrm{W} / \mathrm{m} \cdot \mathrm{K}), \\ \mathrm{d}_{\mathrm{ox2}} & = & \text { thickness of oxide layer }(\mathrm{m}) .\end{array}$

\subsection{Identification of Regions with Double-Sided Oxidation}

The correct modeling of cladding oxidation and meltdown requires a correct identification of the regions in a fuel rod where its cladding is being oxidized on both its inner and outer surfaces. The large degree of test bundle degradation that occurs in severe fuel damage tests limits PIE results from these experiments as a basis for developing an empirical model for identification of the regions with doublesided oxidation. But theoretical analyses ${ }^{7}$, experimental results for loss of coolant accidents, $, 923,24$ and experimental results for the quenching of hot fuel rods ${ }^{25}$ provide a basis for improving the model in SCDAP/RELAP5/MOD3.2 for identifying the regions of a reactor core with double-sided oxidation. The improved model is expected to result in a more wide spread agreement with experimental results of calculated rate of heatup and calculated extent of cladding oxidation.

The current model for identifying the regions with double-sided oxidation is shown in Table 10 . The identification of the region of double-sided oxidation is based on only two parameters, namely the cladding hoop strain and whether the cladding has ruptured. If the cladding has ruptured and the local hoop strain is greater than the user-defined threshold hoop strain $\left(e_{t h}\right)$, then double-sided oxidation is modeled as occurring at that location. Otherwise, the oxidation is considered to be single-sided. The threshold hoop strain for double-sided oxidation has a default value of 0.07 . The current model does not account for the circumferential relocation of the liquefied layer of metallic cladding and its effect on the area on which 
oxidation occurs. The current model also does not account for double-sided oxidation occurring in the event that hot embrittled cladding experiences the beginning of quenching.

Table 10. Current model for identifying regions with double-sided oxidation.

\begin{tabular}{|c|c|c|}
\hline $\begin{array}{c}\text { Local } \\
\text { cladding } \\
\text { hoop strain }\end{array}$ & $\begin{array}{c}\text { Cladding } \\
\text { rupture? }\end{array}$ & $\begin{array}{c}\text { Number of } \\
\text { sides of } \\
\text { oxidation }\end{array}$ \\
\hline \hline$<e_{t h}$ & No & $I$ \\
\hline$<e_{t h}$ & $Y e s$ & $I$ \\
\hline$>e_{t h}$ & No & $I$ \\
\hline$>e_{t h}$ & $Y e s$ & 2 \\
\hline
\end{tabular}

The proposed model will determine the regions of double-sided oxidation using different parameters than the current model. Instead of evaluating the ratio of local hoop strain to a threshold hoop strain, the distance a location is from a breach in the cladding will be compared with the steam penetration distance, represented by the symbol $l_{i o}$. If a location is within $l_{\text {io }}$ of a breach in the cladding, then double-sided oxidation is assumed to occur at that location. Theoretical results ${ }^{6}$ for the case of large cladding hoop strains and experimental results ${ }^{9,23}$ indicate that the value of $l_{i o}$ is only a few $\mathrm{cm}$. Thus, $\mathrm{l}_{\mathrm{io}}$ will be assumed to have a value of 0.05 . The relatively small value for $1_{\mathrm{io}}$ is in part due to the $\mathrm{Zr}$ within a few $\mathrm{cm}$ of the breach being able to consume all of the available steam and in part due to the expansion of the gases inside the fuel-cladding gap as the temperature rises and as further fission gas release occurs. The value of $l_{\text {io }}$ may be greater under reflood conditions but the increase in value will be assumed to be small relative to the length of an axial node, which in general is $0.10 \mathrm{~m}$ in length or greater.

Experiments have shown that a breach due to ballooning in the cladding during severe accident conditions may extend over a considerable length compared to that for large break loss of coolant accidents $^{2,26}$. In the PBF SFD 1-1 test, ${ }^{2}$ cladding breach and double-sided oxidation were observed over a length of $0.4 \mathrm{~m}$ and within this $0.4 \mathrm{~m}$ length the cladding ballooned to hoop strains of about 0.18 or greater. Since a mechanistic model for calculating the length of cladding breach is not available and the length of cladding breach in severe accident tests is usually not available due to the cladding damage that occurs during the experiments, the length of breach will be defined to be the portion of the cladding extending upward and downward from the location of initial cladding rupture with a hoop strain greater than 0.18 . Double-sided oxidation will be calculated to occur at any location within the axial section of the cladding with a breach.

The identification of the region of a fuel rod with double-sided oxidation will take into account whether the metallic part of the cladding relocates in the circumferential direction after it liquefies. As previously shown in Figure 14, circumferential relocation is assumed to cause the inner surface of the metallic layer to be in contact with fuel and thus blocked from contact with steam. So for the case of circumferential relocation of liquefied cladding, oxidation will be assumed to occur only on the outside surface of the metallic layer. 
If hot cladding is locally embrittled and in the process of being quenched, double-sided oxidation occurs at that location due to cracks that develop in the cladding and result in paths for steam to contact the inner surface of the cladding. ${ }^{25}$ The model for identifying the regions with double-sided oxidation will be extended to account for this experimentally observed behavior of the cladding. The model will apply variables calculated by SCDAP/RELAP5/MOD3.2 to determine whether the cladding is locally embrittled and whether it is locally being subjected to quenching. In addition to the existing model for calculating embrittlement of the cladding, which calculates embrittlement based only upon oxygen uptake, the new model for identifying the region of double-sided oxidation will apply the embrittlement of the cladding due to hydrogen uptake ${ }^{8,25}$. This model for cladding embrittlement is defined in another preliminary design report ${ }^{20}$. If the cladding at an axial node is locally embrittled and in the process of being quenched, then double-sided oxidation will be defined to occur at that axial node.

The proposed model for identifying regions of the reactor core with double-sided oxidation is summarized in Table 11. The table has a column for each of the four variables in the model for identifying the region of double-sided oxidation; (1) indicator of cladding rupture, (2) distance from breach in cladding, (3) indicator of melting of liquefied part of cladding, and (4) indicator of cladding being quenched after embrittlement. Column entries that are blank denote variables that do not influence whether the oxidation is single-sided or double-sided for the values shown in the other columns for that row of the table. Double-sided oxidation occurs at a locality only when two states of the cladding exist; (1) the locality is within $l_{\text {io }}$ of a cladding breach and the local metallic part of the cladding is not liquefied, and (2) the locality is embrittled, in the process of being quenched, and the local metallic part of the cladding is not liquefied. The case of the simultaneous occurrence of a liquefied metallic layer and quenching is not shown in this table because for this state of the cladding the oxide layer is expected to crack and release the liquefied material it retained.

Table 11. Proposed model for identifying regions with double-sided oxidation.

\begin{tabular}{|c|c|c|c|c|}
\hline $\begin{array}{c}\text { cladding } \\
\text { rupture? }\end{array}$ & $\begin{array}{c}\text { Distance } \\
\text { from } \\
\text { breach }\end{array}$ & $\begin{array}{c}\text { Metallic } \\
\text { cladding } \\
\text { liquefied? }\end{array}$ & $\begin{array}{c}\text { Cladding } \\
\text { embrittled } \\
\text { and } \\
\text { quench } \\
\text { occurring? }\end{array}$ & $\begin{array}{c}\text { Number } \\
\text { of sides of } \\
\text { oxidation }\end{array}$ \\
\hline \hline no & $(\mathrm{m})$ & no & yes & 2 \\
\hline yes & & $y e s$ & no & 1 \\
\hline yes & $>l_{\text {io }}$ & & no & 1 \\
\hline yes & $<l_{\text {io }}$ & no & & 2 \\
\hline
\end{tabular}




\section{Integration and Implementation}

This section describes the integration and implementation of models for the configuration and integrity of partially oxidized cladding at high temperatures. The models to be integrated and implemented were described in Section 4 of this report. The described models perform calculations to determine; (1) configuration of metallic part of cladding after it has liquefied, (2) effect of relocated material on integrity of oxidic part of cladding, (3) thickness of oxidic part of cladding accounting for oxidation and dissolution, (4) stress in oxidic part of cladding relative to its failure stress, and (5) locations of cladding with doublesided oxidation.

The five models for cladding configuration and integrity supply information for each other and for other damage progression models. The integration of the five models and the application of their output are shown in Figure 18 , Figure 19, and Figure 20. These figures are flow charts of the order in which various

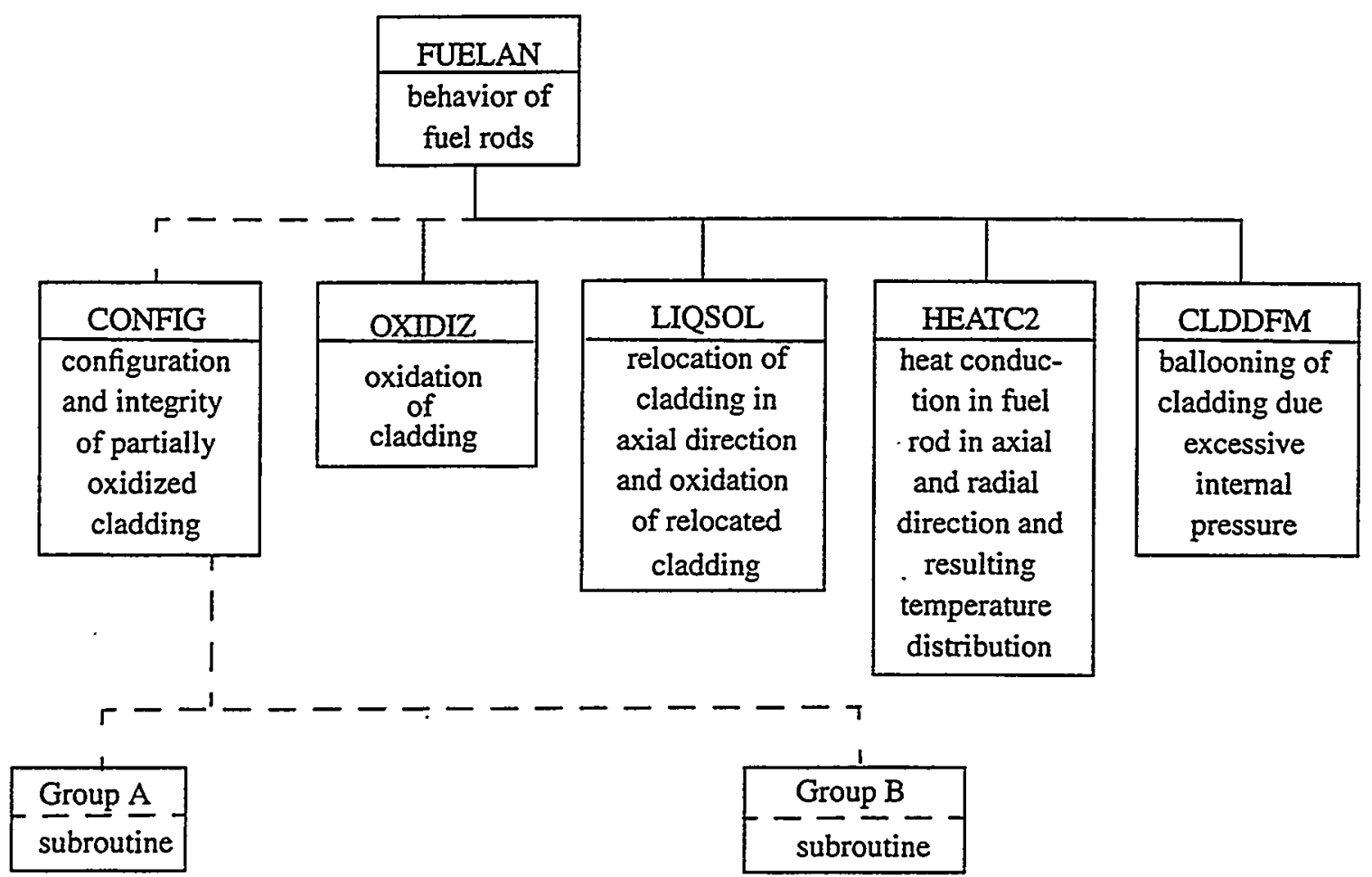

Figure 18. Integration and application of models for cladding configuration and integrity.

subroutines in SCDAP/RELAP5 are called to calculate the progression of damage in fuel rods. The five models for cladding configuration and integrity are each represented by a new subroutine. The sequence of all capitalized letters at the top of a box in the figure is the name of the subroutine represented by that box. The function of each subroutine is also summarized in the box representing the subroutine. The solid lines connecting subroutines indicate subroutines that currently exist and are implemented. The dashed lines connecting subroutines indicate the implementation of subroutines that contain the models described in this report. For these subroutines, the input variables and the source of the input variables are identified. Also, the output variables of these subroutines are identified. As shown in Figure 16, the major function of the five subroutines will be to feed information to the subroutines that calculate cladding oxidation 


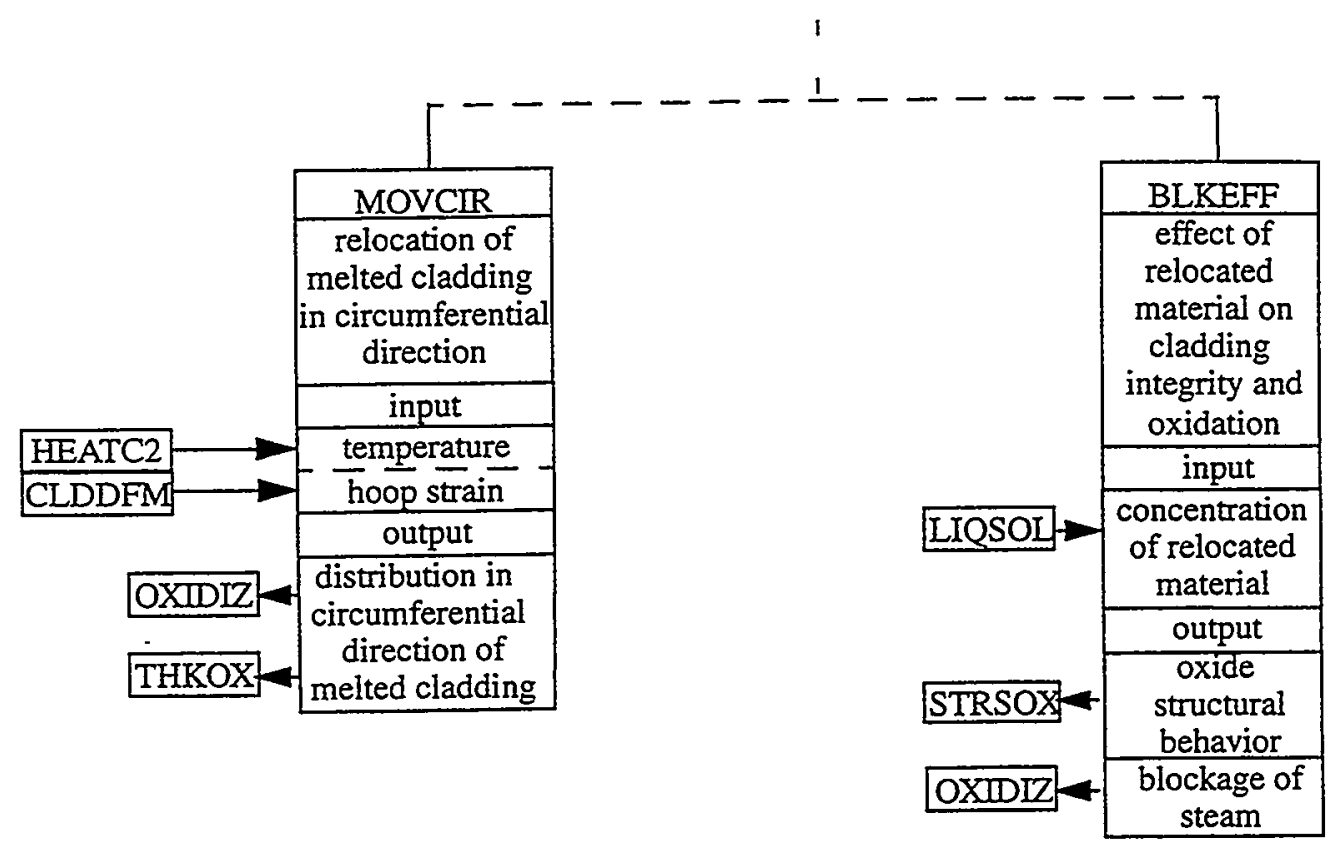

Figure 19. Flow chart of Group A subroutines in Figure 18.

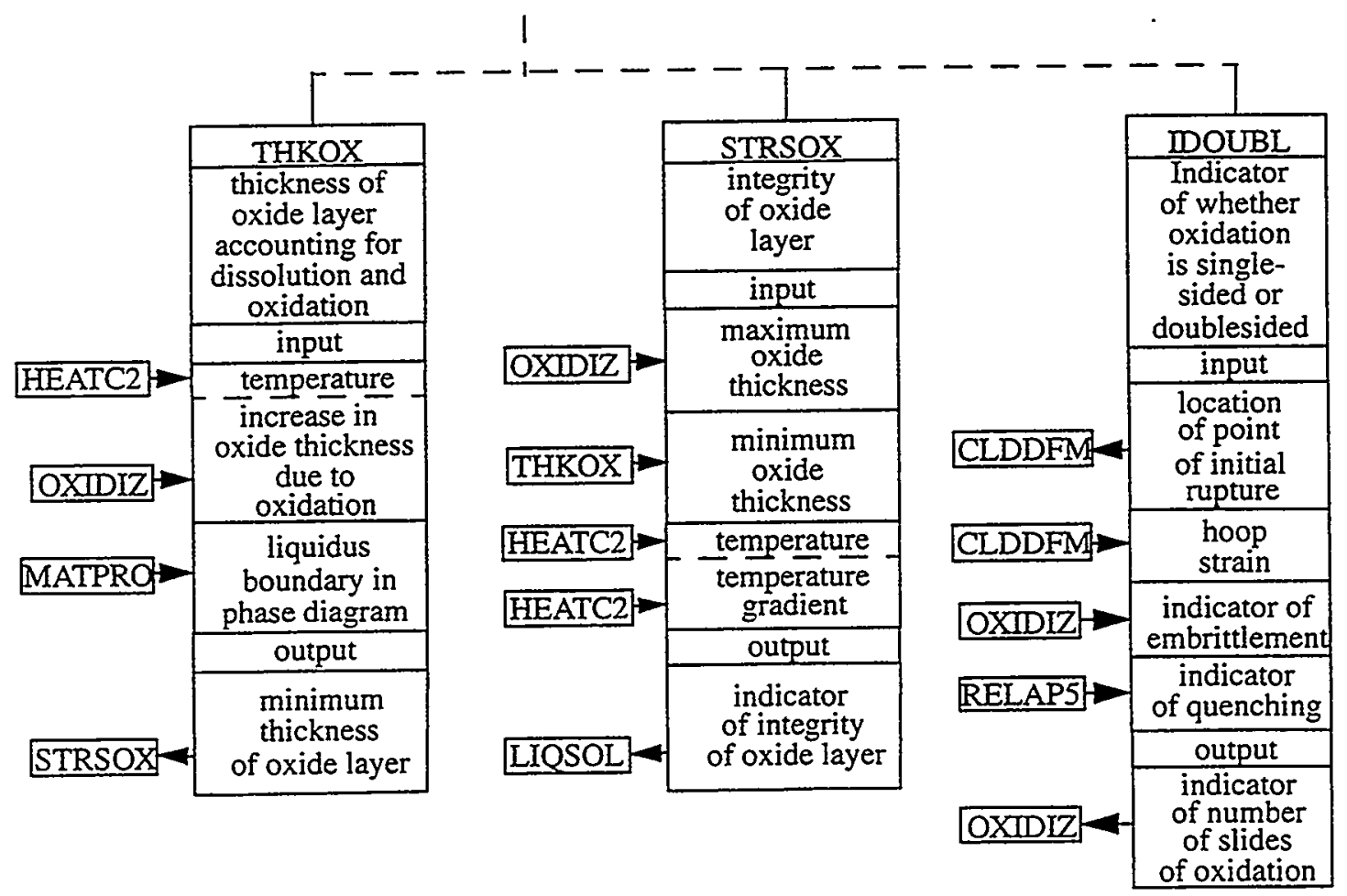

Figure 20. Flow chart of Group B subroutines in Figure 18. 
(OXIDIZ) and cladding relocation in the axial direction (LIQSOL). Cladding oxidation will be calculated taking into account the reduction in surface area (about 40\%) that occurs when melted cladding relocates in the circumferential direction. It will also be calculated taking into account the effect of relocated material on oxidation as indicated by PIE results. The locations with double-sided oxidation of the cladding will be identified taking into account theoretical and experimental results. The integrity of the cladding oxide layer and the time at which it fails and releases the retained melted cladding will be calculated based on a structural analysis of the oxide layer. The integrity of the oxide layer will be calculated by the new subroutine named STRSOX, which replaces the calculation in the existing subroutine named ZOFAIL.

\section{Testing and Assessment of Model Improvements}

The modeling improvements described in Sections 3 through 7 will be tested and assessed using the results of the integral experiments presented in Section 2 and using the results of other test problems used in the developmental assessment of SCDAP/RELAP5/MOD3.2..$^{1}$ The calculation results that will be compared with experimental results are; (1) axial variation in extent of oxidation of cladding, (2) total hydrogen production, (3) axial variation in amount of the local relocated material, (4) axial variation in rate of cladding heatup, and (5) axial variation in the local maximum fuel rod temperatures. If modeling improvements result in a closer agreement of calculation results with experimental results for each of these five areas of experimental results, then the modeling improvements are qualified for implementation into the code. The test matrix to be executed and the improvements expected in calculation results are shown in Table 12. In order to assess the impact of the modeling improvements on the results of full-plant calculations, a calculation will also be performed of the Surry TMLB' accident scenario used in the developmental assessment of SCDAP/RELAP5/MOD3.21.

Table 12. Matrix of test problems for assessing improvements in modeling of cladding oxidation and meltdown.

\begin{tabular}{|c|c|c|}
\hline $\begin{array}{c}\text { Problem } \\
\text { No. }\end{array}$ & $\begin{array}{l}\text { Problem } \\
\text { name }\end{array}$ & Areas of calculations where improvements are expected \\
\hline 1 & PBF SFD 1-1 & $\begin{array}{l}\text { 1. Increase fraction of cladding oxidation at } 0.23 \mathrm{~m} \text { elev. from } 0.13 \text { to } 1.0 \\
\text { 2. Decrease fraction of clad. oxidation at } 0.74 \mathrm{~m} \text { elev. from } 0.46 \text { to } 0.10 \\
\text { 3. Increase max. temperature at } 0.14 \mathrm{~m} \text { elevation from } 1800 \mathrm{~K} \text { to } 2890 \mathrm{~K} \\
\text { 4. Max. blockage in } 0.05 \text { to } 0.20 \mathrm{~m} \text { elev. instead of } 0.30 \text { to } 0.40 \mathrm{~m} \text { elev. }\end{array}$ \\
\hline 2 & PBF SFD 1-4 & $\begin{array}{l}\text { 1. Increase fraction of cladding oxidation at } 0.15 \mathrm{~m} \text { elev. from } 0.02 \text { to } 1.0 \\
\text { 2. Decrease fraction of clad. oxidation at } 0.85 \mathrm{~m} \text { elev. from } 0.86 \text { to } 0.22 \\
\text { 3. Increase max. temperature at } 0.15 \mathrm{~m} \text { elev. from } 1500 \mathrm{~K} \text { to }>2000 \mathrm{~K} \\
\text { 4. } 100 \% \text { instead of } 43 \% \text { blockage in elev. interval of } 0.20 \mathrm{~m} \text { to } 0.30 \mathrm{~m}\end{array}$ \\
\hline 3 & $\begin{array}{c}K f K \text { CORA- } \\
13\end{array}$ & $\begin{array}{l}\text { 1. Increase fraction of clad. oxidation at } 0.45 \mathrm{~m} \text { elev. from } 0.10 \text { to } 1.0 \\
\text { 2. Decrease frac. of clad. oxid. in } 0.80 \text { to } 1.0 \mathrm{~m} \text { elev. from } 0.40 \text { to }<0.05 \\
\text { 3. Decrease max. temperature at } 0.25 \mathrm{~m} \text { from } 2500 \mathrm{~K} \text { to } 1530 \mathrm{~K} \\
\text { 4. } 100 \% \text { instead of } 55 \% \text { blockage in elev. interval of } 0.4 \mathrm{~m} \text { to } 0.5 \mathrm{~m}\end{array}$ \\
\hline
\end{tabular}


Table 12. Matrix of test problems for assessing improvements in modeling of cladding oxidation and meltdown. (Continued)

\begin{tabular}{|c|c|c|}
\hline $\begin{array}{c}\text { Problem } \\
\text { No. }\end{array}$ & $\begin{array}{l}\text { Problem } \\
\text { name }\end{array}$ & Areas of calculations where improvements are expected \\
\hline 4 & $\begin{array}{c}\text { PHEBUS } \\
B 9_{+}\end{array}$ & 1. Decrease fraction of clad. oxidation at $0.41 \mathrm{~m}$ elev. from 0.86 to 0.40 \\
\hline 5 & $\begin{array}{c}O E C D \\
L O F T \text { FP-2 }\end{array}$ & $\begin{array}{l}\text { 1. Decrease fraction of oxidation at } 0.48 \mathrm{~m} \text { from } 0.90 \text { to }<0.05 \\
\text { 2. Increase max. temperature at } 1.20 \mathrm{~m} \text { elev. from } 2200 \mathrm{~K} \text { to } 2330 \mathrm{~K} \\
\text { 3. } 100 \% \text { block. in } 0.66-0.77 \mathrm{~m} \text { elev. instead of } 11 \% \text { block. in } 0.85-0.09 \mathrm{~m}\end{array}$ \\
\hline 6 & FLHT-5 & 1. Increase elev. of max. block. from interval of $0.8-1.0 \mathrm{~m}$ to $1.2-1.8 \mathrm{~m}$ \\
\hline 7 & $P B F S F D-S T$ & 1. Increase blockage in $0.15 \mathrm{~m}$ to $0.20 \mathrm{~m}$ elev. interval from 0.0 to $100 \%$ \\
\hline 8 & $K f K C O R A-7$ & 1. Decrease elev. of max. block. from interval of $0.7-0.8 . m$ to $0.35-0.50 \mathrm{~m}$ \\
\hline 9 & KfK CORA-5 & 1. Increase elev. of max. block. from interval of $0.15-0.25 \mathrm{~m}$ to $0.40-0.5 \mathrm{~m}$ \\
\hline 10 & $\begin{array}{c}\text { PHEBUS } \\
\text { FPTO }\end{array}$ & 1. Increase total hydrogen production from $70 \%$ to $100 \%$ of measured value \\
\hline 11 & $\begin{array}{c}\text { TMI-2 acci- } \\
\text { dent }\end{array}$ & 1. Decrease elevation of bottom of molten region from $1.46 \mathrm{~m}$ to $0.71 \mathrm{~m}$ \\
\hline 12 & Surry TMLB' & 1. No areas for improvement are identified \\
\hline
\end{tabular}

\section{Summary}

Models have been designed for resolving deficiencies in the SCDAP/RELAP5/MOD3.2 calculations of the behavior of hot, partially oxidized cladding. These deficiencies were identified from comparisons of calculated and measured variations in the axial direction of the extent of cladding oxidation and the extent of fuel rod relocation. In order to resolve the deficiencies, models were designed for several different aspects of fuel rod behavior. In general, the modeling changes are empirical rather than mechanistic due to mechanistic modeling requiring an order of magnitude increase in the spatial detail that describes fuel rod behavior and due to material properties for mechanistic modeling requiring material properties that are not firmly established. First, an improved mapping was established from a compilation of PIE results from severe fuel damage tests of the configuration of melted metallic cladding that is retained by an oxide layer. The improved mapping accounts for the relocation of melted cladding in the circumferential direction. Rules were established for calculating the effect of relocated cladding on the oxidation and integrity of the intact cladding upon which it solidifies. Next, two different methods were presented for calculating the extent of dissolution of the oxidic part of the cladding due to its contact with the metallic part. The extent of dissolution effects the stress and thus the integrity of the oxidic part of the cladding. Then, an empirical equation was presented for calculating the stress in the oxidic part of the cladding and evaluating its integrity based on this calculated stress. This empirical equation replaces the current criterion for loss of integrity which is based on temperature and extent of oxidation. Finally, a new rule based on theoretical and experimental results was established for identifying the regions of a fuel rod with oxidation of both the inside and outside surfaces of the cladding. After integration of each of these modeling changes, the 
calculated axial distribution in cladding oxidation and relocation is expected to be in better agreement with experimental results than is currently the case.

The modeling improvements will be assessed first of all by comparisons of calculated and measured axial distributions in cladding oxidation. While the assessment of SCDAP/RELAP/MOD3.2 indicated a good agreement between calculated and measured rates of heatup and total hydrogen production, the assessment performed in this report indicated that the extent of oxidation was generally overpredicted in the upper part of test fuel rod bundles and was generally underpredicted in the lower part of the test fuel bundles and in the region with a large concentration of relocated material. Therefore, the first step of the assessment effort will be to verify that these deficiencies have been resolved by the modeling improvements. Then, comparisons of calculations and experimental results will also be performed to verify that the modeling changes have maintained a good modeling capability for the other areas of severe fuel damage progression, such as rate of heatup and total hydrogen production. Since the heatup caused by hydrogen uptake in the upper portion of a bundle of fuel rods may be of the same order of magnitude as that caused by oxidation and thus may have a strong influence on the calculation of severe fuel damage progression, it is recommended that the assessment of the modeling improvements not be performed until a model for hydrogen uptake and corresponding heatup has been implemented into the code.

\section{References}

1. The SCDAP/RELAP5 Development Team, "SCDAP/RELAP5/MOD3.2 Code Manual, Volume V; Developmental Assessment," NUREG/CR-6150, INEL-96/0422, Revision 1, October 1997.

2. Z. R. Martinson, D.A. Petti, and B. A. Cook, "Vol. 1: PBF Severe Fuel Damage Test 1-1 Test Results Report," NUREG/CR-4684, EGG-2463, Volume 1, October 1986.

3. D. A. Petti et al, "Power Burst Facility (PBF) Severe Fuel Damage Test 1-4 Test Results Report," NUREG/CR-5163, EGG-2542 R3, April, 1989.

4. S. Hagen et al, "Results of SFD Experiment CORA-13 (OECD International Standard Problem 31)," KfK 5054, Kernforschungszentrum Karlsruhe, February 1993.

5. B. Adroguer, S. Bourdon, and R. Gonzalez, "Analysis of Fuel-Cladding Interaction in PHEBUS SFD Tests Using ICARE2 Code," IAEA Technical Committee Meeting "Behaviour of Core Materials and Fission Product Release in Accident Conditions in Light Water Reactors," CEN Cadarache, March 1619, 1992.

6. S. M. Jensen, D. W. Akers, and B. A. Pregger, "Postirradiation Examination Data and Analyses for OECD LOFT Fission Product Experiment LP-FP-2," Volume 1, OECD LOFT-T-3810, December 1989.

7. D. R. Olander, "Materials chemistry and transport modeling for severe accident analyses in light-water reactors; II: Gap processes and heat release," Nuclear Engineering and Design 148 (1994), pages 273292.

8. A. M. Voltchek and A. V. Nikolaeva, "Hydrogen Interaction with Zircaloy Cladding at High Temperatures," NSI-SARR-32-96, Russian Academy of Sciences, Nuclear Safety Institute, July 1996.

9. I. Catton, J. O. Cermak, Y. S. Kuo, and A. R. Wazzan, "An evaluation of several aspects of the hypothetical loss-of-coolant accident for light water reactors," University of California at Los Angeles, Rep. UCLA-ENG-7450, 1974. 
10. The SCDAP/RELAP5 Development Team, "SCDAP/RELAP5/MOD3.2 Code Manual, Volume 2 (Rev. 1); Damage Progression Model Theory," NUREG/CR-6150, INEL-96/0422, Revision 1, October 1997.

11. B. Adroguer, S. Bourdon, and R. Gonzalez, "Analysis of Fuel-Cladding Interaction in PHEBUS SFD Tests Using ICARE2 Code," IPSN, CEA, IAEA Technical Committee Meeting, CEN Cadarache, March 16-19, 1992.

12. Akihide Hidaka et al., "Influence of thermal properties of zirconia shroud on analysis of PHEBUS FPTO bundle degradation test with ICARE2 code," Nuclear Engineering and Design 168 (1997), 361371.

13. Fumiya Tanabe and Ken Muramatsu, "Thermal-hydraulics in Uncovered Core of Light Water Reactor in Severe Core Damage Accident," Journal of Nuclear Science and Technology, 23[9], 779-793.

14. M. S. Veshchunov and A. V. Palagin, "Investigation of downward relocation of molten materials" Journal of Nuclear Materials 252 (1998) 110-120.

15. Aida N. Wilhelm and Eduardo A. Garcia, "Simulation of the Dissolution Kinetics of $\mathrm{ZrO}_{2}$ by Molten Zircaloy-4 between 2000 and 2400 C," Journal of Nuclear Materials 171 (1990) JNM01007.

16. M. S. Veshchunov and A. V. Berdyshev, "Simultaneous dissolution of $\mathrm{UO}_{2}$ and $\mathrm{ZrO}_{2}$ by molten $\mathrm{Zr}$ in an oxidizing atmosphere," Journal of Nuclear Materials 252 (1998) 98-109.

17. P. Hofmann et. al., "Mechanishes and chemishes Verhalten von Zircaloy-4 Hullrohren and $\mathrm{UO}_{2}$ Brennstoff bei hohen Temperaturen," KfK 4100, Kemforschungszentrum Karlsruhe (1987).

18. D. R. Olander, "Materials chemistry and transport modeling for severe accident analyses in light-water reactors - I: External cladding oxidation," Nuclear Engineering and Design, 148 (1994) 253-271

19. The SCDAP/RELAP5 Development Team, "SCDAP/RELAP5/MOD3.2 Code Manual, Volume 4 (Rev. 1); MATPRO - A Library of Materials Properties for Light-Water-Reactor Accident Analysis," NUREG/CR-6150, INEL-96/0422, Revision 1, October 1997.

20. L. J. Siefken, "Preliminary Design Report for Modeling of Hydrogen Uptake in Fuel Rod Cladding during Severe Accidents," INEEL/EXT-98-00664 (August 1998).

21. R. Viskanta, R.R. Hobbins, and H. Esmaili, "Peer Review of the Modeling of Hydrogen Uptake in Fuel Rod Cladding during Severe Accidents," ER/NRC 98-204 (October 1998).

22. A. Boldyrev at al., "Fuel Cladding Deformation Behavior Module (CROX)," NSI-SARR-05-94, Russian Academy of Sciences, Nuclear Safety Institute, 1994.

23. T. Furuta, H. Uetsuka, and S. Kawasaki, "Estimation of conservatism of present embrittlement criteria for Zircaloy fuel cladding under LOCA," Zirconium for the Nuclear Industry, Sixth Int. Symp., ASTM Spec. Tech Publ. 824 (1984) 734.

24. E. D. Hindle and C. A. Mann, "An experimental study of the deformation of Zircaloy PWR fuel rod cladding under mainly convective cooling," Zirconium in the Nuclear Industry, Fifth Int. Symp., ASTM Spec. Tech. Publ. 754 (1982) 284.

25. P. Hofmann et al., "Experiments on the Quench Behavior of LWR Fuel Rod Segments," Third International QUENCH Workshop, Karlsruhe, Germany, December 2 - 4, 1997.

26. J. M. Broughton et. al., "PBF LOCA Test Series Report, Tests LOC-3 and LOC-5 Fuel Behavior Report," NUREG/CR-2073, EGG-2094 (June 1981). 